\title{
A Trust Region Method Based on Interior Point Techniques for Nonlinear Programming
}

\author{
Richard H. Byrd $^{*} \quad$ Jean Charles Gilbert ${ }^{\dagger} \quad$ Jorge Nocedal $^{\ddagger}$
}

August 10, 1998

\begin{abstract}
An algorithm for minimizing a nonlinear function subject to nonlinear inequality constraints is described. It applies sequential quadratic programming techniques to a sequence of barrier problems, and uses trust regions to ensure the robustness of the iteration and to allow the direct use of second order derivatives. This framework permits primal and primal-dual steps, but the paper focuses on the primal version of the new algorithm. An analysis of the convergence properties of this method is presented.
\end{abstract}

Key words: constrained optimization, interior point method, large-scale optimization, nonlinear programming, primal method, primal-dual method, SQP iteration, barrier method, trust region method.

\footnotetext{
${ }^{*}$ Computer Science Department, University of Colorado, Boulder CO 80309. This author was supported by NSF grant CCR-9101795, ARO grant DAAH04-94-0228, and AFOSR grant F49620-94-1-0101.

${ }^{\dagger}$ INRIA Rocquencourt, B.P. 105, 78153 Le Chesnay Cedex (France).

${ }^{\ddagger}$ ECE Department, Northwestern University, Evanston Il 60208. This author was supported by National Science Foundation Grants CCR-9400881 and ASC-9213149, and by Department of Energy Grant DEFG02-87ER25047-A004.
} 


\section{Introduction}

Sequential Quadratic Programming (SQP) methods have proved to be very efficient for solving medium-size nonlinear programming problems $[12,11]$. They require few iterations and function evaluations, but since they need to solve a quadratic subproblem at every step, the cost of their iteration is potentially high for problems with large numbers of variables and constraints. On the other hand, interior-point methods have proved to be very successful in solving large linear programming problems, and it is natural to ask whether they can be extended to nonlinear problems. Preliminary computational experience with simple adaptations of primal-dual interior point methods have given encouraging results on some classes on nonlinear problems (see for example [27, 14, 29, 1]).

In this paper we describe and analyze an algorithm for large-scale nonlinear programming that uses ideas from interior point methods and sequential quadratic programming. One of its unique features is the use of a trust region framework that allows for the direct use of second derivatives and the inaccurate solution of subproblems. The algorithm is well suited for handling equality constraints (see [4]), but for simplicity of exposition we will only consider here inequality constrained problems of the form

$$
\begin{array}{rl}
\min _{x} & f(x) \\
\text { subject to } & g(x) \leq 0,
\end{array}
$$

where $f: \mathbf{R}^{n} \rightarrow \mathbf{R}$ and $g: \mathbf{R}^{n} \rightarrow \mathbf{R}^{m}$ are smooth functions.

Following the strategy of interior point methods (see for example [13, 28, 19]) we associate with (1.1) the following barrier problem in the variables $x$ and $s$

$$
\begin{array}{rl}
\min _{x, s} & f(x)-\mu \sum_{i=1}^{m} \ln s^{(i)} \\
\text { subject to } & g(x)+s=0,
\end{array}
$$

where $\mu>0$ and where the vector of slack variables $s=\left(s^{(1)}, \ldots, s^{(m)}\right)^{\top}$ is implicitly assumed to be positive.

The main goal of this paper is to propose and analyze an algorithm for finding an approximate solution to (1.2), for fixed $\mu$, that can effectively enforce the positivity condition $s>0$ on the slack variables without incurring in a high cost. This algorithm can be applied repeatedly to problem (1.2), for decreasing values of $\mu$, to approximate the solution of the original problem (1.1). The key to our approach is to view interior point methods from the perspective of sequential quadratic programming and formulate the quadratic subproblem so that the steps are discouraged from violating the bounds $s>0$. This framework suggests how to generate steps with primal or primal-dual characteristics, and is well suited for large problems. Numerical experiments with an implementation of the new method have been performed by Byrd, Hribar and Nocedal [4], and show that this approach holds much promise. We should note that in this paper we do not address the important issue of how fast to decrease the barrier parameter, which is currently an active area of research. 
We begin by introducing some notation and by stating the first-order optimality conditions for the barrier problem. The Lagrangian of (1.2) is

$$
L(x, s, \lambda)=f(x)-\mu \sum_{i=1}^{m} \ln s^{(i)}+\lambda^{\top}(g(x)+s),
$$

where $\lambda \in \mathbf{R}^{m}$ are the Lagrange multipliers. At an optimal solution $(x, s)$ of (1.2) we have

$$
\begin{gathered}
\nabla_{x} L(x, s, \lambda)=\nabla f(x)+A(x) \lambda=0 \\
\nabla_{s} L(x, s, \lambda)=-\mu S^{-1} e+\lambda=0
\end{gathered}
$$

where

$$
A(x)=\left(\nabla g^{(1)}(x), \ldots, \nabla g^{(m)}(x)\right)
$$

is the matrix of constraint gradients, and where

$$
e=\left(\begin{array}{c}
1 \\
\vdots \\
1
\end{array}\right), \quad S=\left(\begin{array}{ccc}
s^{(1)} & & \\
& \ddots & \\
& & s^{(m)}
\end{array}\right)
$$

To facilitate the derivation of the new algorithm we define

$$
\begin{gathered}
z=\left(\begin{array}{c}
x \\
s
\end{array}\right), \quad \varphi(z)=f(x)-\mu \sum_{i=1}^{m} \ln s^{(i)}, \\
c(z)=g(x)+s,
\end{gathered}
$$

and rewrite the barrier problem (1.2) as

$$
\begin{aligned}
\min _{z} & \varphi(z) \\
\text { subject to } & c(z)=0 .
\end{aligned}
$$

We now apply the sequential quadratic programming method (see for example $[12,11]$ ) to this problem. At an iterate $z$, we generate a displacement

$$
d=\left(\begin{array}{c}
d_{x} \\
d_{s}
\end{array}\right)
$$

by solving the quadratic program

$$
\begin{aligned}
\min _{d} & \nabla \varphi(z)^{\top} d+\frac{1}{2} d^{\top} W d \\
\text { subject to } & \hat{A}(z)^{\top} d+c(z)=0,
\end{aligned}
$$

where $W$ is the Hessian of the Lagrangian of the barrier problem (1.10) with respect to $z$, and where $\hat{A}^{\top}$ is the Jacobian of $c$ and is given by

$$
\hat{A}(z)^{\top}=\left(A(x)^{\top} I\right) .
$$


Note that (1.10) is just a restatement of (1.2), and thus from (1.4)-(1.5) we have that

$$
W \equiv \nabla_{z z}^{2} L(x, s, \lambda)=\left(\begin{array}{cc}
\nabla_{x x}^{2} L(x, s, \lambda) & 0 \\
0 & \mu S^{-2}
\end{array}\right) .
$$

To obtain convergence from remote starting points, and to allow for the case when $W$ is not positive definite in the null space of $\hat{A}^{\top}$, we introduce a trust region constraint in (1.11) of the form

$$
\left\|\left(\begin{array}{c}
d_{x} \\
S^{-1} d_{s}
\end{array}\right)\right\| \leq \Delta,
$$

where the trust region radius $\Delta>0$ is updated at every iteration. The step in the slack variables is scaled by $S^{-1}$ due to the form $\mu S^{-2}$ of the portion of the Hessian $W$ corresponding to the slack variables. Since this submatrix is positive definite and diagonal, it seems to be the best scale at the current point; see also [4] for a discussion of how this scaling is beneficial when using a conjugate gradient iteration to compute the step.

From now on we simplify the notation by writing a vector such as $z$, which has $x$ and $s$-components, as $z=(x, s)$ instead of $z=\left(x^{\top}, s^{\top}\right)^{\top}$. In this way an expression like that in (1.14) is simply written as

$$
\left\|\left(\begin{array}{c}
d_{x} \\
S^{-1} d_{s}
\end{array}\right)\right\| \equiv\left\|\left(d_{x}, S^{-1} d_{s}\right)\right\| .
$$

The trust region constraint (1.14) does not prevent the new slack variable values $s+d_{s}$ from becoming negative unless $\Delta$ is sufficiently small. Since it is not desirable to impede progress of the iteration by employing small trust regions, we explicitly bound the slack variables away from zero by imposing the well-known fraction to the boundary rule [28]

$$
s+d_{s} \geq(1-\tau) s,
$$

where the parameter $\tau \in(0,1)$ is chosen close to 1 . This results in the subproblem

$$
\begin{array}{cl}
\min _{d} & \nabla \varphi(z)^{\top} d+\frac{1}{2} d^{\top} W d \\
\text { subject to } & \hat{A}(z)^{\top} d+c(z)=0 \\
& \left\|\left(d_{x}, S^{-1} d_{s}\right)\right\| \leq \Delta \\
& d_{s} \geq-\tau s
\end{array}
$$

We will assume for simplicity that the trust region is defined using the Euclidean norm, although our analysis would be essentially the same for any other fixed norm. It is true that problem (1.16) could be quite difficult to solve exactly, but we intend to only compute approximate solutions using techniques such as a dogleg method or the conjugate gradient algorithm. Due to the formulation of our subproblem these techniques will tend to avoid the boundaries of the constraints $s>0$ and will locate an approximate solution with moderate cost. To see that our subproblem (1.16) is appropriate, note that if the slack variables are scaled by $S^{-1}$, the feasible region of the transformed problem has the essential 
characteristics of a trust region: it is bounded and contains a ball centered at $z$ whose radius is bounded below by a value that depends on $\Delta$ and not on $z$.

It is well known [26] that the constraints in (1.16) can be incompatible since the steps $d$ satisfying the linear constraints may not lie within the trust region. Several strategies have been proposed to make the constraints consistent [7,6, 24], and in this paper we follow the approach of Byrd [3] and Omojokun [20], which we have found suitable for solving large problems [18].

The strategy of Byrd and Omojokun consists of first taking a normal (or transversal) step $v$ that lies well inside the trust region and that attempts to satisfy the linear constraints in (1.16) as well as possible. To compute the normal step $v$, we choose a contraction parameter $0<\xi<1$ (say $\xi=0.8$ ) that determines a tighter version of the constraints (1.16), i.e., a smaller trust region radius $\xi \Delta$ and tighter lower bounds $-\xi \tau$. Then we approximately solve the problem

$$
\begin{aligned}
\min _{v} & \left\|\hat{A}(z)^{\top} v+c(z)\right\| \\
\text { subject to } & \left\|\left(v_{x}, S^{-1} v_{s}\right)\right\| \leq \xi \Delta \\
& v_{s} \geq-\xi \tau s,
\end{aligned}
$$

where here, and for the rest of the paper, $\|\cdot\|$ denotes the Euclidean (or $\ell_{2}$ ) norm. The normal step $v$ determines how well the linear constraints in (1.16) will be satisfied. We now compute the total step $d$ by approximately solving the following modification of (1.16)

$$
\begin{array}{cl}
\min _{d} & \nabla \varphi(z)^{\top} d+\frac{1}{2} d^{\top} W d \\
\text { subject to } & \hat{A}(z)^{\top} d=\hat{A}(z)^{\top} v \\
& \left\|\left(d_{x}, S^{-1} d_{s}\right)\right\| \leq \Delta \\
& d_{s} \geq-\tau s .
\end{array}
$$

The constraints for this subproblem are always consistent; for example $d=v$ is feasible. Lalee, Nocedal and Plantenga [18] describe direct and iterative methods for approximately solving (1.18) when the number of variables is large.

We now need to decide if the trial step $d$ obtained from (1.18) should be accepted, and for this purpose we introduce a merit function for the barrier problem (1.10). (Recall that our objective at this stage is to solve the barrier problem for a fixed value of the barrier parameter $\mu$.) We follow Byrd and Omojokun and define the merit function to be

$$
\phi(z ; \nu)=\varphi(z)+\nu\|c(z)\|,
$$

where $\nu>0$ is a penalty parameter. Since the Euclidean norm in the second term is not squared, this merit function is non-differentiable. It is also exact in the sense that if $\nu$ is greater than a certain threshold value, then a Karush-Kuhn-Tucker point of the barrier problem (1.2) is a stationary point of the merit function $\phi$. The step $d$ is accepted if it gives sufficient reduction in the merit function; otherwise it is rejected.

We complete the iteration by updating the trust region radius $\Delta$ according to standard trust region techniques that will be discussed later on.

We summarize the discussion given so far by presenting a broad outline of the new algorithm for solving the nonlinear programming problem (1.1). 


\section{Algorithm Outline}

Choose an initial barrier parameter $\mu>0$ and an initial iterate $z=(x, s)$ and Lagrange multipliers $\lambda$.

1. If (1.1) is solved to the required accuracy, stop.

2. Compute and approximate solution of the barrier problem (1.10), as follows.

Choose an initial trust region radius $\Delta>0$, a contraction parameter $\xi \in(0,1)$, and a penalty parameter $\nu>0$ for the merit function (1.19).

(a) If the barrier problem (1.10) is solved to the required accuracy, go to 3 .

(b) Compute a normal step $v=\left(v_{x}, v_{s}\right)$ by approximately solving the normal subproblem

$$
\begin{aligned}
\min _{v} & \left\|\hat{A}(z)^{\top} v+c(z)\right\| \\
\text { subject to } & \left\|\left(v_{x}, S^{-1} v_{s}\right)\right\| \leq \xi \Delta \\
& v_{s} \geq-\xi \tau s .
\end{aligned}
$$

(c) Compute the total step $d=\left(d_{x}, d_{s}\right)$ by approximately solving the tangential subproblem

$$
\begin{array}{cl}
\min _{d} & \nabla \varphi(z)^{\top} d+\frac{1}{2} d^{\top} W d \\
\text { subject to } & \hat{A}(z)^{\top} d=\hat{A}(z)^{\top} v \\
& \left\|\left(d_{x}, S^{-1} d_{s}\right)\right\| \leq \Delta \\
& d_{s} \geq-\tau s .
\end{array}
$$

(d) If the step $d$ does not give a sufficient reduction in the merit function (1.19), decrease $\Delta$ and go to (b). Otherwise, set $x \leftarrow x+d_{x}, s \leftarrow s+d_{s}, z=(x, s)$, compute new Lagrange multipliers $\lambda$, and go to (a).

3. Decrease the barrier parameter $\mu$ and go to 1 .

Since the inequality constraints are already being handled as equalities, this algorithm can be easily extended to handle equality constraints. In that case the nonlinear constraints in (1.10) have the form

$$
c(z)=\left(\begin{array}{c}
g_{\mathrm{E}}(x) \\
g_{\mathrm{I}}(x)+s
\end{array}\right)=0 .
$$

The Jacobian matrix $\hat{A}$ then takes the form

$$
\hat{A}(z)^{\top}=\left(\begin{array}{cc}
A_{\mathrm{E}}(x)^{\top} & 0 \\
A_{\mathrm{I}}(x)^{\top} & I
\end{array}\right),
$$

where $A_{\mathrm{E}}$ and $A_{\mathrm{I}}$ denote the matrices of constraint gradients corresponding to $g_{\mathrm{E}}$ and $g_{\mathrm{I}}$; see [4] for a detailed discussion on the treatment of equality constraints in our new method. 
In $\S 2$ we discuss in more detail when to accept or reject a step, and how to update the trust region. This will allow us to give a complete description of the algorithm. We now digress to discuss the relationship between our approach and other interior point methods. This discussion makes use of the well-known fact that Sequential Quadratic Programming, in at least one formulation, is equivalent to Newton's method applied to the optimality conditions of a nonlinear program [11].

\section{$1.1 \quad$ KKT systems}

The KKT conditions for the equality constrained barrier problem (1.2) give rise to the following system of nonlinear equations in $x, s, \lambda$ (see (1.4), (1.5))

$$
\left(\begin{array}{c}
\nabla f(x)+A(x) \lambda \\
-\mu S^{-1} e+\lambda \\
g(x)+s
\end{array}\right)=0
$$

Applying Newton's method to this system we obtain the iteration

$$
\left(\begin{array}{ccc}
\nabla_{x x}^{2} L & 0 & A(x) \\
0 & \mu S^{-2} & I \\
A(x)^{\top} & I & 0
\end{array}\right)\left(\begin{array}{c}
d_{x} \\
d_{s} \\
\lambda^{+}
\end{array}\right)=\left(\begin{array}{c}
-\nabla f(x) \\
\mu S^{-1} e \\
-g(x)-s
\end{array}\right),
$$

where $\lambda^{+}=\lambda+d_{\lambda}$, and where we have omitted the argument of $\nabla_{x x}^{2} L(x, s, \lambda)$ for brevity. Note that the current values of the multipliers $\lambda$ only enter in (1.23) through $\nabla_{x x}^{2} L$. When the objective function and constraints are linear, we have that $\nabla_{x x}^{2} L=0$, and thus the step does not depend on the current values of these multipliers; for this reason a method based on (1.23) is referred to as a primal interior point method.

Let us now suppose that the quadratic subproblem (1.11) is strictly convex, i.e., that $W$ is positive definite on the null space of $\hat{A}(z)^{\top}$. Then it is easy to see that the solution of (1.11) coincides with the step generated by (1.23). Therefore the SQP approach (1.11) with $W$ given by (1.13) is equivalent to a primal interior point iteration on the barrier subproblem, under the convexity assumption just stated. Several researchers, including Yamashita [27] have noted this relationship.

It is also possible to establish a correspondence between primal-dual interior point methods and the SQP approach. Let us multiply the second row of (1.22) by $S$ to obtain the system

$$
\left(\begin{array}{c}
\nabla f(x)+A(x) \lambda \\
S \lambda-\mu e \\
g(x)+s
\end{array}\right)=0
$$

This may be viewed as a modified KKT system for the inequality constrained problem (1.1), since the second row is a relaxation of the complementary slackness condition (which is obtained when $\mu=0$ ). In the linear programming case, primal-dual methods are based on iteratively solving (1.24) in $x, s, \lambda$. Applying Newton's method to (1.24), and then 
symmetrizing the coefficient matrix by multiplying the second block of equations by $S^{-1}$, results in the iteration

$$
\left(\begin{array}{ccc}
\nabla_{x x}^{2} L & 0 & A(x) \\
0 & S^{-1} \Lambda & I \\
A(x)^{\top} & I & 0
\end{array}\right)\left(\begin{array}{c}
d_{x} \\
d_{s} \\
\lambda^{+}
\end{array}\right)=\left(\begin{array}{c}
-\nabla f(x) \\
\mu S^{-1} e \\
-g(x)-s
\end{array}\right)
$$

where we have defined

$$
\Lambda=\operatorname{diag}\left(\lambda^{(1)}, \ldots, \lambda^{(m)}\right) .
$$

Now the current value of $\lambda$ influences the step through the matrix $\Lambda$ and through $\nabla_{x x}^{2} L$. We refer to (1.25) as the primal-dual iteration.

Consider now the SQP subproblem (1.11) with the Hessian of the Lagrangian $W$ replaced by

$$
\tilde{W}=\left(\begin{array}{cc}
\nabla_{x x}^{2} L(x, s, \lambda) & 0 \\
0 & S^{-1} \Lambda
\end{array}\right) .
$$

It is easy to see that if the quadratic program (1.11) is strictly convex, the step generated by the SQP approach coincides with the solution of (1.25). Comparing (1.13) and (1.27) we see that the only difference between the primal and primal-dual SQP formulations is that the matrix $\mu S^{-2}$ has been replaced by $S^{-1} \Lambda$.

This degree of generality justifies the investigation of SQP as a framework for designing interior point methods for nonlinear programming. Several choices for the Hessian matrix $W$ could be considered, but in this study we focus on the (primal) exact Hessian version (1.13) because of its simplicity. We note, however, that much of our analysis could be extended to the primal-dual approach based on (1.27) if appropriate safeguards are applied.

Many authors, among them Panier, Tits, and Herskovits [21], Yamashita [27], Herskovits [15], Anstreicher and Vial [2], Jarre and Saunders [17], El-Bakry, Tapia, Tsuchiya, and Zhang [10], Coleman and Li [8], Dennis, Heinkenschloss and Vicente [9], have proposed interior point methods for nonlinear programming based on iterations of the form (1.23) or (1.25). In some of these studies $\nabla_{x x}^{2} L$ is either assumed positive definite on the whole space or a subspace, or is modified to be so. In our approach there is no such requirement; we can either use the exact Hessian of the Lagrangian with respect to $x$ in (1.23) and (1.25), or any approximation $B$ to it. For example, $B$ could be updated by the BFGS or SR1 quasi-Newton formulae. This generality is possible by the trust region framework described in the previous section.

Plantenga [22] describes an algorithm that has some common features with the algorithm presented here, but his approach has also important differences. Among these are the fact that his trust region does not include a scaling, that his iteration produces affine scaling steps near the solution, and that his approach reverts to an active set method when progress is slow.

We emphasize that the equivalence between SQP and Newton's method applied to the KKT system holds only if the subproblem (1.16) is strictly convex, if this subproblem is solved exactly, and if the trust region constraint is inactive. Since these conditions will not hold in most iterations of our algorithm, the approach presented in this paper is distinct from those based on directly solving the KKT system of the barrier problem. However, 
as the iterates converge to the solution, our algorithm will be very similar to these other interior point methods. This is because near the solution point, the quadratic subproblem (1.11) will be convex and the tolerances of the procedure for solving (1.11) subject to the trust region constraint, will be set so that, asymptotically, it is solved exactly [4]. Moreover, as the iterates converge to the solution we expect the trust region constraint to become inactive, provided a second order correction is incorporated in the algorithm.

In summary the local behavior of our method is similar to that of other interior point methods, but its global behavior is likely to be markedly different. For this reason the analysis presented in this paper will focus on the global convergence properties of the new method.

Notation. Throughout the paper $\|\cdot\|$ denotes the Euclidean (or $\ell_{2}$ ) norm. The vector of slack variables at the $k$-th iteration is written as $s_{k}$, and its $i$-th component is $s_{k}^{(i)}$.

\section{Algorithm for the Barrier Problem}

We now give a detailed description of the algorithm for solving the barrier problem (1.10), that was loosely described in step 2 of the Algorithm Outline in $\S 1$.

From now on we will let $B_{k}$ stand for $\nabla_{x x}^{2} L\left(x_{k}, s_{k}, \lambda_{k}\right)$ or for a symmetric matrix approximating this Hessian. At an iterate $\left(x_{k}, s_{k}\right)$, the step $d$ generated by the algorithm will be an approximate solution of the tangential problem (1.21). Due to the definitions $(1.8),(1.12)$ and $(1.13)$ we can write this tangential problem as

$$
\begin{array}{cl}
\min _{d} & \nabla f_{k}^{\top} d_{x}-\mu e^{\top} S_{k}^{-1} d_{s}+\frac{1}{2} d_{x}^{\top} B_{k} d_{x}+\frac{1}{2} \mu d_{s}^{\top} S_{k}^{-2} d_{s} \\
\text { s.t. } & A_{k}^{\top} d_{x}+d_{s}=A_{k}^{\top} v_{x}+v_{s} \\
& \left\|\left(d_{x}, S_{k}^{-1} d_{s}\right)\right\| \leq \Delta_{k} \\
& d_{s} \geq-\tau s_{k} .
\end{array}
$$

Here, $\nabla f_{k}=\nabla f\left(x_{k}\right)$, and $v$ is the approximate solution to (1.20).

Now we focus on the merit function and, in particular, on how much it is expected to decrease at each iteration. The merit function (1.19) may be expressed as

$$
\phi(x, s ; \nu)=f(x)+\nu\|g(x)+s\|-\mu \sum_{i=1}^{m} \ln s^{(i)} .
$$

We can construct a model $m_{k}$ of $\phi\left(\cdot, \cdot ; \nu_{k}\right)$ around an iterate $\left(x_{k}, s_{k}\right)$ using the quadratic objective from (2.1) and a linear approximation of the constraints in (1.2),

$$
\begin{aligned}
m_{k}(d)= & f_{k}+\nabla f_{k}^{\top} d_{x}+\frac{1}{2} d_{x}^{\top} B_{k} d_{x}+\nu_{k}\left\|g_{k}+s_{k}+A_{k}^{\top} d_{x}+d_{s}\right\| \\
& -\mu\left(\sum_{i=1}^{m} \ln s_{k}^{(i)}+e^{\top} S_{k}^{-1} d_{s}-\frac{1}{2} d_{s}^{\top} S_{k}^{-2} d_{s}\right)
\end{aligned}
$$


We will show in Lemma 3.1 below that $m_{k}$ is a suitable local model of $\phi$. We define the predicted reduction in the merit function $\phi$ to be the change in the model $m_{k}$ produced by a step $d$,

$$
\begin{aligned}
\operatorname{pred}_{k}(d)= & m_{k}(0)-m_{k}(d) \\
= & -\nabla f_{k}^{\top} d_{x}-\frac{1}{2} d_{x}^{\top} B_{k} d_{x} \\
& +\nu_{k}\left(\left\|g_{k}+s_{k}\right\|-\left\|g_{k}+s_{k}+A_{k}^{\top} d_{x}+d_{s}\right\|\right) \\
& +\mu\left(e^{\top} S_{k}^{-1} d_{s}-\frac{1}{2} d_{s}^{\top} S_{k}^{-2} d_{s}\right) .
\end{aligned}
$$

We will always choose the weight $\nu_{k}$ sufficiently large that $\operatorname{pred}_{k}(d)>0$, as will be described in $\S 2.3$.

The predicted reduction is used as a standard for accepting the step and for updating the trust region. We choose a parameter $\eta \in(0,1)$, and if

$$
\phi\left(x_{k}+d_{x}, s_{k}+d_{s} ; \nu_{k}\right) \leq \phi\left(x_{k}, s_{k} ; \nu_{k}\right)-\eta \operatorname{pred}_{k}(d),
$$

we accept the step $d$ and possibly increase the trust region radius $\Delta_{k}$; otherwise we decrease $\Delta_{k}$ by a constant fraction, e.g. $\Delta_{k} \leftarrow \Delta_{k} / 2$, and recompute $d$. Since $\operatorname{pred}_{k}(d)>0$ this implies that the merit function decreases at each step. More sophisticated strategies for updating $\Delta_{k}$ are useful in practice, but this simple rule will be sufficient for our purposes.

Next we consider conditions that determine when approximate solutions to the normal and tangential subproblems are acceptable. Since these conditions require detailed justification, we consider these subproblems separately.

\subsection{Computation of the normal step}

At each step of the algorithm for the barrier problem we first solve the normal subproblem (1.20), which can be written as

$$
\begin{array}{cl}
\min _{v} & \left\|g_{k}+s_{k}+A_{k}^{\top} v_{x}+v_{s}\right\| \\
\text { s.t. } & \left\|\left(v_{x}, S_{k}^{-1} v_{s}\right)\right\| \leq \tilde{\Delta}_{k} \\
& v_{s} \geq-\xi \tau s_{k},
\end{array}
$$

where we have defined

$$
\tilde{\Delta}_{k}=\xi \Delta_{k}
$$

We now present two conditions that an approximate solution $v_{k}$ of (2.6) must satisfy. To do this we introduce the change of variables

$$
u_{x}=v_{x}, \quad u_{s}=S_{k}^{-1} v_{s},
$$

so that problem (2.6) becomes

$$
\begin{array}{cl}
\min _{u} & \left\|g_{k}+s_{k}+A_{k}^{\top} u_{x}+S_{k} u_{s}\right\| \\
\text { s.t. } & \left\|\left(u_{x}, u_{s}\right)\right\| \leq \tilde{\Delta}_{k} \\
& u_{s} \geq-\xi \tau .
\end{array}
$$


In the case where the lower bound constraints are inactive it is straightforward to show [18] that (2.9) has a solution in the range of

$$
\left(\begin{array}{c}
A_{k} \\
S_{k}
\end{array}\right)
$$

Even when the lower bounds are active, keeping $u$ in the range of (2.10) will prevent $u$ from being unreasonably long, and in the implementation of the new method described in [4], $u$ is chosen always in this space. A condition of this type is necessary since, if $u$ is unnecessarily long, the objective function value could get worse, making the job of the tangential step more difficult. For the analysis in this paper it suffices to impose the following weaker condition.

Range Space Condition. The approximate solution $v_{k}$ of the normal problem (2.6) must be of the form

$$
v_{k}=\left(\begin{array}{c}
A_{k} \\
S_{k}^{2}
\end{array}\right) w_{k}
$$

for some vector $w_{k} \in \mathbf{R}^{m}$, whenever (2.6) has an optimal solution of that form.

The second condition on the normal step requires that the reduction in the objective of (2.6) be comparable to that obtained by minimizing along the steepest descent direction in $u$. This direction is the gradient of the objective in problem (2.9) at $u=0$, which is a multiple of

$$
u_{k}^{c} \equiv-\left(\begin{array}{c}
A_{k} \\
S_{k}
\end{array}\right)\left(g_{k}+s_{k}\right)
$$

Transforming back to the original variables we obtain the vector

$$
v_{k}^{c} \equiv-\left(\begin{array}{c}
A_{k} \\
S_{k}^{2}
\end{array}\right)\left(g_{k}+s_{k}\right),
$$

which we call the scaled steepest descent direction. We refer to the reduction in the objective of $(2.6)$ produced by a step $v=\left(v_{x}, v_{s}\right)$ as the normal predicted reduction:

$$
\operatorname{vpred}_{k}(v)=\left\|g_{k}+s_{k}\right\|-\left\|g_{k}+s_{k}+A_{k}^{\top} v_{x}+v_{s}\right\|,
$$

and we require that this reduction satisfy the following condition.

Normal Cauchy Decrease Condition. An approximate solution $v_{k}$ of the normal problem (2.6) must satisfy

$$
\operatorname{vpred}_{k}\left(v_{k}\right) \geq \gamma_{v} \operatorname{vpred}_{k}\left(\alpha_{k}^{c} v_{k}^{c}\right),
$$

for some constant $\gamma_{v}>0$, where $\alpha_{k}^{c}$ solves the problem

$$
\begin{aligned}
\min _{\alpha \geq 0} & \left\|g_{k}+s_{k}+\alpha\left(A_{k}^{\top} v_{x}^{c}+v_{s}^{c}\right)\right\| \\
\text { s.t. } & \left\|\alpha\left(v_{x}^{c}, S_{k}^{-1} v_{s}^{c}\right)\right\| \leq \tilde{\Delta}_{k} \\
& \alpha v_{s}^{c} \geq-\xi \tau s_{k} .
\end{aligned}
$$


Note that the normal Cauchy decrease condition and the range space condition (2.11) are satisfied by an optimal solution of (2.6) with $\gamma_{v} \leq 1$. Both conditions are also satisfied if the step is chosen by truncated conjugate gradient iterations in the variable $u$ on the objective of (2.9) (see Steihaug [25]), and the results are transformed back into the original variables. Also, since $\alpha=0$ is a feasible solution of (2.16), it is clear from (2.15) that

$$
\operatorname{vpred}_{k}\left(v_{k}\right) \geq 0 \text {. }
$$

In Lemma 2.2 we give a sharper bound on the normal predicted reduction $\operatorname{vpred}_{k}\left(v_{k}\right)$ of an approximate solution that satisfies the normal Cauchy decrease condition. First we will find it useful to establish this generalization of the one-dimensional version of a result by Powell [23].

Lemma 2.1. Consider the one dimensional problem

$$
\begin{aligned}
\min _{z \geq 0} & \psi(z) \equiv \frac{1}{2} a z^{2}-b z \\
\text { s.t. } & z \leq t,
\end{aligned}
$$

where $b \geq 0$ and $t>0$. Then the optimal value $\psi_{*}$ satisfies

$$
\psi_{*} \leq-\frac{b}{2} \min \left\{t, \frac{b}{|a|}\right\} \text {. }
$$

Proof. Consider first the case when $a>0$. Then $\frac{b}{a} \geq 0$ is the unconstrained minimizer of $\psi$. If $\frac{b}{a} \leq t$, then the unconstrained minimizer solves the problem and

$$
\psi_{*}=\psi\left(\frac{b}{a}\right)=-\frac{b^{2}}{2 a}
$$

On the other hand, if $\frac{b}{a} \geq t$, since $\psi$ is decreasing on $\left[0, \frac{b}{a}\right]$ and $a t \leq b$,

$$
\psi_{*}=\psi(t)=\frac{1}{2} a t^{2}-b t \leq-\frac{b t}{2} .
$$

In the case $a \leq 0, \psi$ is concave everywhere so that

$$
\psi_{*}=\psi(t) \leq-b t .
$$

Since one of (2.18), (2.19) or (2.20) must hold, the result follows.

Applying this to the normal problem yields the following result.

Lemma 2.2. Suppose that $s_{k}>0$ and that $v_{k}=\left(v_{x}, v_{s}\right)$ is an approximate solution of (2.6) satisfying the normal Cauchy decrease condition (2.15). Then

$$
\begin{gathered}
\left\|g_{k}+s_{k}\right\| \operatorname{vpred}_{k}\left(v_{k}\right)=\left\|g_{k}+s_{k}\right\|\left(\left\|g_{k}+s_{k}\right\|-\left\|g_{k}+s_{k}+A_{k}^{\top} v_{x}+v_{s}\right\|\right) \\
\geq \frac{\gamma_{v}}{2}\left\|\left(\begin{array}{c}
A_{k} \\
S_{k}
\end{array}\right)\left(g_{k}+s_{k}\right)\right\| \min \left(\tilde{\Delta}_{k}, \xi \tau, \frac{\left\|\left(\begin{array}{c}
A_{k} \\
S_{k}
\end{array}\right)\left(g_{k}+s_{k}\right)\right\|}{\left\|\left(A_{k}^{\top} S_{k}\right)\right\|^{2}}\right),
\end{gathered}
$$

where $\gamma_{v}$ is defined in (2.15). 
Proof. Inequality (2.21) clearly holds when $u_{k}^{c}=0$ because (2.12) implies that the right hand side of the inequality is zero. Therefore, we now assume that $u_{k}^{c} \neq 0$. of

By the normal Cauchy decrease condition, (2.8) and (2.12), the scalar $\alpha_{k}^{c}$ is a solution

$$
\begin{array}{ll}
\min _{\alpha \geq 0} & \frac{\alpha^{2}}{2}\left\|\left(A_{k}^{\top} S_{k}\right) u_{k}^{c}\right\|^{2}-\alpha\left\|u_{k}^{c}\right\|^{2} \\
\text { s.t. } & |\alpha| \leq \frac{\tilde{\Delta}_{k}}{\left\|u_{k}^{c}\right\|} \\
& \alpha \leq-\frac{\xi \tau}{\left(u_{s}^{c}\right)^{(i)}} \quad \text { for all } i \text { such that }\left(u_{s}^{c}\right)^{(i)}<0 .
\end{array}
$$

Note that the upper bounds of problem (2.22) are satisfied if

$$
\alpha \leq \min \left\{\frac{\tilde{\Delta}_{k}}{\left\|u_{k}^{c}\right\|}, \frac{\xi \tau}{\left\|u_{k}^{c}\right\|}\right\} .
$$

Using this and Lemma 2.1 we have,

$$
\begin{aligned}
& \frac{1}{2}\left(\left\|g_{k}+s_{k}+\alpha_{k}^{c}\left(A_{k}^{\top} v_{x}^{c}+v_{s}^{c}\right)\right\|^{2}-\left\|g_{k}+s_{k}\right\|^{2}\right) \\
& \quad=\frac{\left(\alpha^{c}\right)^{2}}{2}\left\|\left(A_{k}^{\top} S_{k}\right) u_{k}^{c}\right\|^{2}-\alpha^{c}\left\|u_{k}^{c}\right\|^{2} \\
& \leq-\frac{\left\|u_{k}^{c}\right\|^{2}}{2} \min \left\{\frac{\min \left\{\tilde{\Delta}_{k}, \xi \tau\right\}}{\left\|u_{k}^{c}\right\|}, \frac{\left\|u_{k}^{c}\right\|^{2}}{\left\|\left(A_{k}^{\top} S_{k}\right) u_{k}^{c}\right\|^{2}}\right\} \\
& \quad \leq-\frac{\left\|u_{k}^{c}\right\|}{2} \min \left\{\tilde{\Delta}_{k}, \xi \tau, \frac{\left\|u_{k}^{c}\right\|}{\left\|\left(A_{k}^{\top} S_{k}\right)\right\|^{2}}\right\} .
\end{aligned}
$$

Now, since the normal Cauchy decrease condition holds, by (2.14) and (2.15),

$$
\begin{aligned}
\left\|g_{k}+s_{k}\right\| \operatorname{vpred}_{k}\left(v_{k}\right) & \geq \gamma_{v}\left\|g_{k}+s_{k}\right\|\left(\left\|g_{k}+s_{k}\right\|-\left\|g_{k}+s_{k}+\alpha_{k}^{c}\left(A_{k}^{\top} v_{x}^{c}+v_{s}^{c}\right)\right\|\right) \\
& \geq \frac{\gamma_{v}}{2}\left(\left\|g_{k}+s_{k}\right\|^{2}-\left\|g_{k}+s_{k}+\alpha_{k}^{c}\left(A_{k}^{\top} v_{x}^{c}+v_{s}^{c}\right)\right\|^{2}\right) \\
& \geq \frac{\gamma_{v}}{2}\left\|u_{k}^{c}\right\| \min \left\{\tilde{\Delta}_{k}, \xi \tau, \frac{\left\|u_{k}^{c}\right\|}{\left\|\left(A_{k}^{\top} S_{k}\right)\right\|^{2}}\right\},
\end{aligned}
$$

where we used the inequality $2 a(a-b) \geq a^{2}-b^{2}$. Substituting for $u_{k}^{c}$ by its value given by $(2.12)$, we obtain $(2.21)$.

\subsection{Approximate solution of the tangential problem}

Consider now the tangential subproblem (2.1). Writing $d=v_{k}+h$, where $v_{k}$ is the approximate solution of the normal subproblem, we can write the equality constraints in $(2.1)$ as

$$
A_{k}^{\top} h_{x}+h_{s}=0
$$


It follows from this equation that, if the normal step satisfies (2.11), then the vector $\left(h_{x}, S_{k}^{-1} h_{s}\right)$ is orthogonal to $\left(v_{x}, S_{k}^{-1} v_{s}\right)$. We can therefore write the trust region constraint as

$$
\left\|\left(h_{x}, S_{k}^{-1} h_{s}\right)\right\| \leq \hat{\Delta}_{k},
$$

where

$$
\hat{\Delta}_{k}=\left(\Delta_{k}^{2}-\left\|\left(v_{x}, S_{k}^{-1} v_{s}\right)\right\|^{2}\right)^{\frac{1}{2}} .
$$

If orthogonality does not hold we can still write the trust region constraint as (2.23), where now

$$
\hat{\Delta}_{k}=\Delta_{k}-\left\|\left(v_{x}, S_{k}^{-1} v_{s}\right)\right\| .
$$

This ensures that $d$ is within the trust region of (2.1), although it restricts $h$ more than necessary in some cases. Note from (2.7) that either choice of $\hat{\Delta}_{k}$ implies that

$$
\Delta_{k} \geq \hat{\Delta}_{k} \geq(1-\xi) \Delta_{k}
$$

This and the condition $\left\|\left(d_{x}, S_{k}^{-1} d_{s}\right)\right\| \leq \Delta_{k}$ are the only requirements we place on $\hat{\Delta}_{k}$.

Substituting $d=v_{k}+h$ in (2.1) and omitting constant terms involving $v_{k}$ in the objective function, we obtain the following problem in $h=\left(h_{x}, h_{s}\right)$,

$$
\begin{array}{ll}
\min _{h} & \left(\nabla f_{k}+B_{k} v_{x}\right)^{\top} h_{x}+\frac{1}{2} h_{x}^{\top} B_{k} h_{x} \\
& -\mu\left(e^{\top} S_{k}^{-1} h_{s}-v_{s}^{\top} S_{k}^{-2} h_{s}-\frac{1}{2} h_{s}^{\top} S_{k}^{-2} h_{s}\right) \\
\text { s.t. } & A_{k}^{\top} h_{x}+h_{s}=0 \\
& \left\|\left(h_{x}, S_{k}^{-1} h_{s}\right)\right\| \leq \hat{\Delta}_{k} \\
& S_{k}^{-1}\left(v_{s}+h_{s}\right) \geq-\tau .
\end{array}
$$

We now describe a decrease condition that an approximate solution of (2.25) must satisfy. For this purpose we define the tangential predicted reduction produced by a step $h=\left(h_{x}, h_{s}\right)$ as the change in the objective function of $(2.25)$,

$$
\begin{aligned}
\operatorname{hpred}_{k}(h)= & -\left(\nabla f_{k}+B_{k} v_{x}\right)^{\top} h_{x}-\frac{1}{2} h_{x}^{\top} B_{k} h_{x} \\
& +\mu\left(e^{\top} S_{k}^{-1} h_{s}-v_{s}^{\top} S_{k}^{-2} h_{s}-\frac{1}{2} h_{s}^{\top} S_{k}^{-2} h_{s}\right) .
\end{aligned}
$$

Next, we let $Z_{k}=\left(Z_{x}^{\top} Z_{s}^{\top}\right)^{\top}$ denote a null space basis matrix for the equality constraints in problem $(2.25)$, i.e., $Z_{k}$ is an $(n+m) \times n$ full rank matrix satisfying

$$
\left(A_{k}^{\top} I\right) Z_{k}=A_{k}^{\top} Z_{x}+Z_{s}=0
$$

A simple choice of $Z_{k}$ is to define $Z_{k}=\left(I-A_{k}\right)^{\top}$, but many other choices are possible, and some may have advantages in different contexts. In this paper we will allow $Z_{k}$ to be any null space basis matrix satisfying

$$
\left\|Z_{k}\right\| \leq \gamma_{Z} \quad \text { and } \quad \sigma_{\min }\left(Z_{k}\right) \geq \gamma_{Z}^{-1}, \quad \text { for all } k,
$$


where $\gamma_{Z}$ is a positive constant and $\sigma_{\min }\left(Z_{k}\right)$ denotes the smallest singular value of $Z_{k}$. If $\left\{A_{k}\right\}$ is bounded this condition is satisfied by $Z_{k}=\left(I-A_{k}\right)^{\top}$ and by many other choices of $Z_{k}$.

Any feasible vector for (2.25) may be expressed as $h=Z_{k} p$ for some $p \in \mathbf{R}^{n}$. Thus, writing $h=\left(h_{x}, h_{s}\right)=\left(Z_{x} p, Z_{s} p\right)$, the tangential subproblem (2.25) becomes

$$
\begin{array}{ll}
\min _{p} & \left(\nabla f_{k}+B_{k} v_{x}\right)^{\top} Z_{x} p-\mu\left(S_{k}^{-1} e-S_{k}^{-2} v_{s}\right)^{\top} Z_{s} p \\
& +\frac{1}{2} p^{\top}\left(Z_{x}^{\top} B_{k} Z_{x}+\mu Z_{s}^{\top} S_{k}^{-2} Z_{s}\right) p \\
\text { s.t. } & \left\|\left(Z_{x} p, S_{k}^{-1} Z_{s} p\right)\right\| \leq \hat{\Delta}_{k} \\
& S_{k}^{-1}\left(v_{s}+Z_{s} p\right) \geq-\tau .
\end{array}
$$

Again, this has the form of a trust region subproblem for unconstrained optimization, with bounds at some distance from zero (in the scaled variables) and by analogy with standard practice, we will require that the step $h_{k}=Z_{k} p_{k}$ give as much reduction in the objective of (2.29) as a steepest descent step. The steepest descent direction for the objective function of $(2.29)$ at $p=0$ is given by

$$
p_{k}^{c}=-Z_{x}^{\top}\left(\nabla f_{k}+B_{k} v_{x}\right)+\mu Z_{s}^{\top}\left(S_{k}^{-1} e-S_{k}^{-2} v_{s}\right) .
$$

We are now ready to state the condition we impose on the tangential step.

Tangential Cauchy Decrease Condition. The approximate solution $h_{k}$ of the tangential problem (2.25) must satisfy

$$
\operatorname{hpred}_{k}\left(h_{k}\right) \geq \gamma_{h} \operatorname{hpred}_{k}\left(\theta_{k}^{c} Z_{k} p_{k}^{c}\right),
$$

for some constant $\gamma_{h}>0$, where $\theta_{k}^{c}$ solves the problem

$$
\begin{array}{cl}
\min _{\theta \geq 0} & -\operatorname{hpred}_{k}\left(\theta Z_{k} p_{k}^{c}\right) \\
\text { s.t. } & \left\|\theta\left(Z_{x} p_{k}^{c}, S_{k}^{-1} Z_{s} p_{k}^{c}\right)\right\| \leq \hat{\Delta}_{k} \\
& v_{s}+\theta Z_{s} p_{k}^{c} \geq-\tau s_{k} .
\end{array}
$$

Here $Z_{k}$ is a null space basis matrix satisfying (2.28) and $\hat{\Delta}_{k}$ satisfies (2.24).

The tangential Cauchy decrease condition is clearly satisfied by the optimal solution of (2.29). It is also satisfied if the step is chosen by truncated conjugate gradient iterations in the variable $p$ on the objective of (2.29) (see Steihaug [25]). Note also that since $\theta=0$ is a feasible solution to (2.32),

$$
\operatorname{hpred}_{k}\left(h_{k}\right) \geq 0 \text {. }
$$

The following result establishes a lower bound on the tangential predicted reduction $\operatorname{hpred}_{k}\left(h_{k}\right)$ for a step satisfying the tangential Cauchy decrease condition. 
Lemma 2.3. Suppose that $s_{k}>0$ and that $h_{k}=\left(h_{x}, h_{s}\right)$ satisfies the tangential Cauchy decrease condition (2.31). Then

$$
\operatorname{hpred}_{k}\left(h_{k}\right) \geq \frac{\gamma_{h}}{2}\left\|p_{k}^{c}\right\| \min \left(\frac{\min \left(\hat{\Delta}_{k},(1-\xi) \tau\right)}{\left\|Z_{x}^{\top} Z_{x}+Z_{s}^{\top} S_{k}^{-2} Z_{s}\right\|^{1 / 2}}, \frac{\left\|p_{k}^{c}\right\|}{\left\|Z_{x}^{\top} B_{k} Z_{x}+\mu Z_{s}^{\top} S_{k}^{-2} Z_{s}\right\|}\right),
$$

where $p_{k}^{c}$ is given by (2.30) and $\gamma_{h}$ is used in (2.31).

Proof. Note that the problem (2.32) may be expressed as

$$
\begin{array}{cl}
\min _{\theta \geq 0} & -\operatorname{hpred}_{k}\left(\theta Z_{k} p_{k}^{c}\right)=\frac{1}{2}\left(p_{k}^{c}\right)^{\top}\left(Z_{x}^{\top} B_{k} Z_{x}+\mu Z_{s}^{\top} S_{k}^{-2} Z_{s}\right) p_{k}^{c} \theta^{2}-\left\|p_{k}^{c}\right\|^{2} \theta \\
\text { s.t. } & |\theta| \leq \frac{\hat{\Delta}_{k}}{\left\|\left(Z_{x} p_{k}^{c}, S_{k}^{-1} Z_{s} p_{k}^{c}\right)\right\|} \\
& \theta \leq-\frac{\tau+\left(S_{k}^{-1} v_{s}\right)^{(i)}}{\left(S_{k}^{-1} Z_{s} p_{k}^{c}\right)^{(i)}} \text { for all } i \text { such that }\left(S_{k}^{-1} Z_{s} p_{k}^{c}\right)^{(i)}<0 .
\end{array}
$$

Since the normal problem ensures that $\left(S_{k}^{-1} v_{s}\right)^{(i)} \geq-\xi \tau$, it follows from the definition of the Euclidean norm that the upper bounds on $\theta$ in the last group of (2.35) are greater than or equal to

$$
\frac{(1-\xi) \tau}{\left\|\left(Z_{x} p_{k}^{c}, S_{k}^{-1} Z_{s} p_{k}^{c}\right)\right\|}
$$

Applying Lemma 2.1 to problem (2.35) we then have

$$
\begin{aligned}
- & \operatorname{hpred}_{k}\left(\theta_{k}^{c} Z_{k} p_{k}^{c}\right) \\
& \leq-\frac{1}{2}\left\|p_{k}^{c}\right\|^{2} \min \left\{\frac{\min \left\{\hat{\Delta}_{k},(1-\xi) \tau\right\}}{\left\|\left(Z_{x} p_{k}^{c}, S_{k}^{-1} Z_{s} p_{k}^{c}\right)\right\|}, \frac{\left\|p_{k}^{c}\right\|^{2}}{\left|\left(p_{k}^{c}\right)^{\top}\left(Z_{x}^{\top} B_{k} Z_{x}+\mu Z_{s}^{\top} S_{k}^{-2} Z_{s}\right) p_{k}^{c}\right|}\right\} .
\end{aligned}
$$

The result (2.34) then follows from norm inequalities and (2.31) .

\subsection{Detailed description of the algorithm}

Now that we have specified how the normal and tangential subproblems are to be solved, we can give a precise description of our algorithm for solving the barrier problem (1.2).

Algorithm I. Choose the initial iterate $z_{0}=\left(x_{0}, s_{0}, \lambda_{0}\right)$ with $s_{0}>0$, the initial trust region radius $\Delta_{0}>0$, four constants $\xi, \eta, \rho$, and $\tau$ in $(0,1)$ and a positive constant $\nu_{-1}$. Set $k=0$.

1. Compute the normal step $v_{k}=\left(v_{x}, v_{s}\right)$ by solving approximately (2.6), in such a way that $v_{k}$ satisfies the range space condition (2.11) and the normal Cauchy decrease condition (2.15).

2. Compute the tangent step $h_{k}=\left(h_{x}, h_{s}\right)$ by solving approximately $(2.25)$, in such a way that $h_{k}$ satisfies the tangential Cauchy decrease condition (2.31), and the total step $d_{k}=\left(d_{x}, d_{s}\right)=v_{k}+h_{k}$ satisfies $\left\|\left(d_{x}, S_{k}^{-1} d_{s}\right)\right\| \leq \Delta_{k}$. 
3. Update the penalty parameter of the merit function (2.2) as follows. Let $\tilde{\nu}_{k}$ be the smallest value of $\nu_{k}$, such that

$$
\operatorname{pred}_{k}\left(d_{k}\right) \geq \rho \nu_{k} \operatorname{vpred}_{k}\left(v_{k}\right) .
$$

If $\tilde{\nu}_{k} \leq \nu_{k-1}$, set $\nu_{k}=\nu_{k-1}$; otherwise set $\nu_{k}=\max \left(\tilde{\nu}_{k}, 1.5 \nu_{k-1}\right)$.

4. If

$$
\phi\left(x_{k}+d_{x}, s_{k}+d_{s} ; \nu_{k}\right)>\phi\left(x_{k}, s_{k} ; \nu_{k}\right)-\eta \operatorname{pred}_{k}\left(d_{k}\right)
$$

decrease $\Delta_{k}$ by a constant factor and go to 1 .

5. Set $x_{k+1}=x_{k}+d_{x}, s_{k+1}=\max \left(s_{k}+d_{s},-g_{k+1}\right)$, compute a new multiplier $\lambda_{k+1}$, update $B_{k}$, choose a new value $\Delta_{k+1} \geq \Delta_{k}$, increase $k$ by 1 and go to 1 .

Steps 3 and 5 need some clarification. Writing $d_{x}=h_{x}+v_{x}$ and $d_{s}=h_{s}+v_{s}$, the total predicted reduction (2.4) becomes

$$
\begin{aligned}
& \operatorname{pred}_{k}\left(d_{k}\right)= \\
& \quad-\nabla f_{k}^{\top} v_{x}-\frac{1}{2} v_{x}^{\top} B_{k} v_{x}-\left(\nabla f_{k}+B_{k} v_{x}\right)^{\top} h_{x}-\frac{1}{2} h_{x}^{\top} B_{k} h_{x} \\
& \quad+\nu_{k}\left(\left\|g_{k}+s_{k}\right\|-\left\|g_{k}+s_{k}+A_{k}^{\top} d_{x}+d_{s}\right\|\right) \\
& \quad+\mu\left(e^{\top} S_{k}^{-1} v_{s}-\frac{1}{2} v_{s}^{\top} S_{k}^{-2} v_{s}\right)+\mu\left(e^{\top} S_{k}^{-1} h_{s}-v_{s}^{\top} S_{k}^{-2} h_{s}-\frac{1}{2} h_{s}^{\top} S_{k}^{-2} h_{s}\right) .
\end{aligned}
$$

Recalling the definitions (2.14) and (2.26) of the normal and tangential predicted reductions, we obtain

$$
\operatorname{pred}_{k}\left(d_{k}\right)=\nu_{k} \operatorname{vpred}_{k}\left(v_{k}\right)+\operatorname{hpred}_{k}\left(h_{k}\right)+\chi_{k},
$$

where

$$
\chi_{k}=-\nabla f_{k}^{\top} v_{x}-\frac{1}{2} v_{x}^{\top} B_{k} v_{x}+\mu\left(e^{\top} S_{k}^{-1} v_{s}-\frac{1}{2} v_{s}^{\top} S_{k}^{-2} v_{s}\right) .
$$

We have noted in (2.17) and (2.33) that $\operatorname{vpred}_{k}\left(v_{k}\right)$ and $\operatorname{hpred}_{k}\left(h_{k}\right)$ are both nonnegative, but $\chi_{k}$, which gives the change in the objective of (2.1) due to the normal step $v_{k}$, can be of any sign. Condition (2.36) in step 3 compensates for the possible negativity of this term by choosing a sufficiently large value of $\nu_{k}$, so that $\operatorname{pred}_{k}\left(d_{k}\right)$ is at least a fraction $\rho$ of $\nu_{k} \operatorname{vpred}_{k}\left(v_{k}\right)$. More precisely, from (2.38) we see that if $\operatorname{vpred}_{k}\left(v_{k}\right)>0,(2.36)$ holds when

$$
\nu_{k} \geq \frac{-\chi_{k}}{(1-\rho) \operatorname{vpred}_{k}\left(v_{k}\right)} .
$$

On the other hand, if $\operatorname{vpred}_{k}\left(v_{k}\right)=0$, then by (2.21) and $s_{k}>0$, it must be the case that $g_{k}+s_{k}=0$. In that case $v=0$ is a solution to (2.6) and by the range space condition $v_{k}$ is in the range of $\left(A_{k}^{\top} S_{k}^{2}\right)^{\top}$. Since $s_{k}>0$ the squared objective of (2.6) is a positive definite quadratic on that subspace, so $v=0$ is the unique minimizer in that space. This uniqueness implies that $v_{k}=0$. In that case $\chi_{k}=0$ and (2.36) is satisfied for any value of $\nu_{k}$. 
In step 5 we do not always set $s_{k+1}=s_{k}+d_{s}$, because when $g_{k+1}^{(i)}<0$, the $i$-th constraint is feasible and we have more freedom in choosing the corresponding slack, $s_{k+1}^{(i)}$. In this case our rule ensures that the new slack is not unnecessarily small. Furthermore, it is always the case that $\phi\left(x_{k}+d_{x}, s_{k+1} ; \nu_{k}\right) \leq \phi\left(x_{k}+d_{x}, s_{k}+d_{s} ; \nu_{k}\right)$, so that this update rule for $s_{k+1}$ does not increase the value of the merit function obtained after acceptance of the trust region radius.

Finally note that we have left the strategy for computing the Lagrange multipliers and $B_{k}$ unspecified. The treatment in this paper allows $B_{k}$ to be any bounded approximation to $\nabla_{x x}^{2} L\left(x_{k}, s_{k}, \lambda_{k}\right)$, and allows $\lambda_{k}$ to be any multiplier estimate consistent with this boundedness. The important question of what choices of $B_{k}$ and $\lambda_{k}$ are most effective is not addressed here, and we refer the reader to [4] for some possibilities.

\section{Well-posedness of Algorithm I}

The purpose of this section is to show that, if an iterate $\left(x_{k}, s_{k}\right)$ is not a stationary point of the barrier problem, then the trust region radius cannot shrink to zero and prevent the algorithm from moving away from that point. We begin by showing that $m_{k}$ is an accurate local model of the merit function $\phi$. To analyze this accuracy we define the actual reduction in the merit function $\phi$ from $\left(x_{k}, s_{k}\right)$ to $\left(x_{k}+d_{x}, s_{k}+d_{s}\right)$ as

$$
\operatorname{ared}_{k}(d)=\phi\left(x_{k}, s_{k} ; \nu_{k}\right)-\phi\left(x_{k}+d_{x}, s_{k}+d_{s} ; \nu_{k}\right) .
$$

Step 4 of Algorithm I thus states that a step $d$ is acceptable if

$$
\operatorname{ared}_{k}(d) \geq \eta \operatorname{pred}_{k}(d) .
$$

Lemma 3.1. Suppose that $\nabla f$ and $A$ are Lipschitz continuous on an open convex set $X$ containing all the iterates $\left\{x_{k}\right\}$ generated by Algorithm $I$, and assume that $\left\{B_{k}\right\}$ is bounded. Then there is a positive constant $\gamma_{L}$ such that for any iterate $\left(x_{k}, s_{k}\right)$ and any step $\left(d_{x}, d_{s}\right)$ such that the segment $\left[x_{k}, x_{k}+d_{x}\right]$ is in $X$ and $d_{s} \geq-\tau s_{k}$,

$$
\left|\operatorname{pred}_{k}(d)-\operatorname{ared}_{k}(d)\right| \leq \gamma_{L}\left(\left(1+\nu_{k}\right)\left\|d_{x}\right\|^{2}+\left\|S_{k}^{-1} d_{s}\right\|^{2}\right) .
$$

Proof. Using the Lipschitz continuity of $A$, we have for some positive constant $\gamma^{\prime}$,

$$
\begin{aligned}
& \left|\left\|g\left(x_{k}+d_{x}\right)+s_{k}+d_{s}\right\|-\left\|g_{k}+s_{k}+A_{k}^{\top} d_{x}+d_{s}\right\|\right| \\
& \quad \leq\left\|g\left(x_{k}+d_{x}\right)-g_{k}-A_{k}^{\top} d_{x}\right\| \\
& \quad \leq \sup _{\xi \in\left[x_{k}, x_{k}+d_{x}\right]}\left\|A(\xi)-A_{k}\right\|\left\|d_{x}\right\| \\
& \quad \leq \gamma^{\prime}\left\|d_{x}\right\|^{2} .
\end{aligned}
$$

Similarly, for any scalars $\sigma$ and $\sigma^{\prime}$ satisfying $\sigma>0$ and $\sigma^{\prime} \geq-\tau \sigma$,

$$
\left|\ln \left(\sigma+\sigma^{\prime}\right)-\ln \sigma-\frac{\sigma^{\prime}}{\sigma}\right| \leq \sup _{t \in\left[\sigma, \sigma+\sigma^{\prime}\right]}\left|\frac{\sigma^{\prime}}{t}-\frac{\sigma^{\prime}}{\sigma}\right|=\frac{\sigma}{\sigma+\sigma^{\prime}}\left(\frac{\sigma^{\prime}}{\sigma}\right)^{2} \leq \frac{1}{1-\tau}\left(\frac{\sigma^{\prime}}{\sigma}\right)^{2} .
$$

Using these two inequalities, the definitions (3.1), (2.4) of $\operatorname{ared}_{k}(d)$ and $\operatorname{pred}_{k}(d)$, the Lipschitz continuity of $\nabla f$, and the boundedness of $\left\{B_{k}\right\}$, we have 


$$
\begin{aligned}
& \left|\operatorname{pred}_{k}(d)-\operatorname{ared}_{k}(d)\right| \\
& =\mid f\left(x_{k}+d_{x}\right)-f_{k}-\nabla f_{k}^{\top} d_{x}-\frac{1}{2} d_{x}^{\top} B_{k} d_{x} \\
& \quad+\nu_{k}\left(\left\|g\left(x_{k}+d_{x}\right)+s_{k}+d_{s}\right\|-\left\|g_{k}+s_{k}+A_{k}^{\top} d_{x}+d_{s}\right\|\right) \\
& \quad-\mu \sum_{i=1}^{m}\left(\ln \left(s_{k}+d_{s}\right)^{(i)}-\ln s_{k}^{(i)}-\frac{d_{s}^{(i)}}{s_{k}^{(i)}}+\frac{1}{2}\left(\frac{d_{s}^{(i)}}{s_{k}^{(i)}}\right)^{2}\right) \mid \\
& \leq \gamma^{\prime \prime}\left(1+\nu_{k}\right)\left\|d_{x}\right\|^{2}+\mu\left(\frac{1}{1-\tau}+\frac{1}{2}\right)\left\|S_{k}^{-1} d_{s}\right\|^{2},
\end{aligned}
$$

for some positive constant $\gamma^{\prime \prime}$.

In the next proposition, we show that Algorithm I determines an acceptable step with a finite number of reductions of $\Delta_{k}$, i.e., that there can be no infinite cycling between steps 1 and 4 of Algorithm I. For this it is important that we ensure that, by decreasing the trust region radius, we are able to make the displacement in $s$ arbitrarily small.

Proposition 3.2. Suppose that $s_{k}>0$ and that $\left(x_{k}, s_{k}\right)$ is not a stationary point of the barrier problem (1.2). Then there exists $\Delta_{k}^{0}>0$, such that if $\Delta_{k} \in\left(0, \Delta_{k}^{0}\right)$, the inequality (3.2) holds.

Proof. We proceed by contradiction, supposing that there is a subsequence (indexed by $i$, the iteration counter $k$ is fixed here) of trust region radii $\Delta_{k, i}$ converging to zero, and corresponding steps $d_{k, i}=v_{k, i}+h_{k, i}$ and penalty parameters $\nu_{k, i}$, such that $\operatorname{ared}_{k, i}\left(d_{k, i}\right)<$ $\eta \operatorname{pred}_{k, i}\left(d_{k, i}\right)$ for all $i$.

The inequality $\operatorname{ared}_{k, i}\left(d_{k, i}\right)<\eta \operatorname{pred}_{k, i}\left(d_{k, i}\right)$ and the assumption $\eta \in(0,1)$ imply that $\left|\operatorname{pred}_{k, i}\left(d_{k, i}\right)-\operatorname{ared}_{k, i}\left(d_{k, i}\right)\right|>(1-\eta) \operatorname{pred}_{k, i}\left(d_{k, i}\right)$. This together with the limits $d_{x}^{k, i} \rightarrow 0$, $d_{s}^{k, i} \rightarrow 0$, and Lemma 3.1 gives

$$
\operatorname{pred}_{k, i}\left(d_{k, i}\right)=\left(1+\nu_{k, i}\right) o\left(\left\|d_{x}^{k, i}\right\|\right)+o\left(\left\|d_{s}^{k, i}\right\|\right)
$$

We will show that this equation leads to a contradiction, which will prove the proposition. For the rest of the proof $\gamma_{1}^{\prime}, \gamma_{2}^{\prime}, \ldots$, denote positive constants (independent of $i$ but not of $k$ ), and to simplify the notation, we omit the arguments in $\operatorname{vpred}_{k, i}\left(v_{k, i}\right), \operatorname{hpred}_{k, i}\left(h_{k, i}\right)$, and $\operatorname{pred}_{k, i}\left(d_{k, i}\right)$.

Consider first the case when $g_{k}+s_{k}=0$. From (2.14) and (2.17), we see that $\operatorname{vpred}_{k, i}=$ 0 . Also, since $g_{k}+s_{k}=0,(2.6)$ has a solution $(v=0)$ in the range space of $\left(A_{k}^{\top} S_{k}^{2}\right)^{\top}$, so that the range space condition (2.11) implies that $v_{k, i}$ is of the form (2.11), for some vector $w_{k, i}$. Therefore $0=\operatorname{vpred}_{k, i}=\left\|\left(A_{k}^{\top} A_{k}+S_{k}^{2}\right) w_{k, i}\right\|$, which implies that $w_{k, i}=0$ and $v_{k, i}=0$, because the matrix inside the parenthesis is nonsingular. Given that $\operatorname{vpred}_{k, i}$ and $v_{k, i}$ both vanish, we have from (2.37), (2.38) and (2.33) that $\operatorname{pred}_{k, i}=\operatorname{hpred}_{k, i} \geq 0$. Hence, inequality (2.36) holds independently of the value of $\nu_{k, i}$, implying that $\left\{\nu_{k, i}\right\}_{i \geq 1}$ is bounded. Therefore, (3.4) gives

$$
\operatorname{pred}_{k, i}=o\left(\left\|d_{x}^{k, i}\right\|\right)+o\left(\left\|d_{s}^{k, i}\right\|\right) .
$$


On the other hand, from (2.30) and $v_{k, i}=0$ we see that $p_{k}^{c}=-Z_{x}^{\top} \nabla f_{k}+\mu Z_{s}^{\top} S_{k}^{-1} e$. This vector is nonzero; otherwise the KKT conditions of the barrier problem (1.2) and the definition (2.27) of $Z_{k}$, would imply that $\left(x_{k}, s_{k}\right)$ is a stationary point of the problem. Then, for $\hat{\Delta}_{k, i}$ sufficiently small, inequality (2.34), the trust region in (2.25), and the fact that $h_{k, i}=d_{k, i}$ give

$$
\operatorname{pred}_{k, i}=\operatorname{hpred}_{k, i} \geq \gamma_{1}^{\prime} \hat{\Delta}_{k, i} \geq \gamma_{1}^{\prime}\left\|\left(d_{x}^{k, i}, S_{k}^{-1} d_{s}^{k, i}\right)\right\| \geq \gamma_{2}^{\prime}\left(\left\|d_{x}^{k, i}\right\|+\left\|d_{s}^{k, i}\right\|\right) .
$$

This contradicts (3.5).

Consider now the case when $g_{k}+s_{k} \neq 0$. Since the matrix $\left(A_{k}^{\top} S_{k}\right)$ has full rank, and by $\tilde{\Delta}_{k, i} \rightarrow 0$, we deduce from (2.21) that for $i$ large

$$
\operatorname{vpred}_{k, i} \geq \gamma_{3}^{\prime} \tilde{\Delta}_{k, i}
$$

Then, from step 3 of the algorithm, (3.6), and the fact that $\left\|d_{x}^{k, i}\right\|+\left\|d_{s}^{k, i}\right\| \leq\left(\gamma_{4}^{\prime}\right)^{-1} \tilde{\Delta}_{k, i}$, we obtain

$$
\begin{aligned}
\operatorname{pred}_{k, i} & \geq \rho \nu_{k, i} \operatorname{vpred}_{k, i} \\
& \geq \rho \nu_{k, i} \gamma_{3}^{\prime} \tilde{\Delta}_{k, i} \\
& \geq \rho \nu_{k, i} \gamma_{3}^{\prime} \gamma_{4}^{\prime}\left(\left\|d_{x}^{k, i}\right\|+\left\|d_{s}^{k, i}\right\|\right)
\end{aligned}
$$

Since, $\nu_{k, i} \geq \nu_{-1}>0$ this contradicts (3.4), concluding the proof.

\section{Global Analysis of Algorithm I}

We now analyze the global behavior of Algorithm I when applied to the barrier problem (1.2) for a fixed value of $\mu$. To establish the main result of this section we make the following assumptions about the problem and the iterates generated by the algorithm.

Assumptions 4.1. (a) The functions $f$ and $g$ are differentiable on an open convex set $X$ containing all the iterates $x_{k}$, and $\nabla f, g$, and $A$ are Lipschitz continuous on $X$. (b) The sequence $\left\{f_{k}\right\}$ is bounded below and the sequences $\left\{\nabla f_{k}\right\},\left\{g_{k}\right\},\left\{A_{k}\right\}$ and $\left\{B_{k}\right\}$ are bounded.

Note that we have not assumed that the matrices of constraint gradients $A_{k}$ have full rank because we want to explore how the algorithm behaves in the presence of dependent constraint gradients. Our most restrictive assumption is (b), which could be violated if the iterates are unbounded. The practical value of our analysis, as we will show, is that the situations under which Algorithm I can fail represent problem characteristics that are of interest to a user and that can be characterized in simple mathematical terms. As we proceed with the analysis, we will point out how it makes specific demands on some of the more subtle aspects of Algorithm I whose role may not be apparent to the reader at this point. Therefore the analysis that follows provides a justification for the design of our algorithm. 
We adopt the notation $\alpha^{+}=\max (0, \alpha)$, for a scalar $\alpha$, while for a vector, $u^{+}$is defined component-wise by $\left(u^{+}\right)^{(i)}=\left(u^{(i)}\right)^{+}$. We also make use of the measure of infeasibility $x \mapsto\left\|g(x)^{+}\right\|$, which vanishes if and only if $x$ is feasible for the original problem (1.1). Note that $\left\|g(\cdot)^{+}\right\|^{2}$ is differentiable and has for gradient

$$
\nabla\left\|g(x)^{+}\right\|^{2}=2 A(x) g(x)^{+} .
$$

We make use of the following definitions; here $A^{(i)}$ denotes the $i$-th column of $A$.

Definitions 4.2. A sequence $\left\{x_{k}\right\}$ is asymptotically feasible if $g\left(x_{k}\right)^{+} \rightarrow 0$. We say that the sequence $\left\{\left(g_{k}, A_{k}\right)\right\}$ has a limit point $(\bar{g}, \bar{A})$ failing the linear independence constraint qualification, if the set $\left\{\bar{A}^{(i)}: \bar{g}^{(i)}=0\right\}$ is rank deficient.

Note that the concept of constraint qualification usually applies to a point $x$, but that we extend it to characterize limit points of the sequence $\left\{\left(g_{k}, A_{k}\right)\right\}$, and thus our definition is not standard. The main result we will establish for Algorithm I is the following.

Theorem 4.3. Suppose that Algorithm I is applied to the barrier problem (1.2) and that Assumptions 4.1 hold. Then,

1) the sequence of slack variables $\left\{_{k}\right\}$ is bounded,

2) $A_{k}\left(g_{k}+s_{k}\right) \rightarrow 0$ and $S_{k}\left(g_{k}+s_{k}\right) \rightarrow 0$.

Furthermore, one of the following three situations occurs.

(i) The sequence $\left\{x_{k}\right\}$ is not asymptotically feasible. In this case, the iterates approach stationarity of the measure of infeasibility $x \mapsto\left\|g(x)^{+}\right\|$, meaning that $A_{k} g_{k}^{+} \rightarrow 0$, and the penalty parameters $\nu_{k}$ tend to infinity.

(ii) The sequence $\left\{x_{k}\right\}$ is asymptotically feasible, but the sequence $\left\{\left(g_{k}, A_{k}\right)\right\}$ has a limit point $(\bar{g}, \bar{A})$ failing the linear independence constraint qualification. In this situation also, the penalty parameters $\nu_{k}$ tend to infinity.

(iii) The sequence $\left\{x_{k}\right\}$ is asymptotically feasible and all limit points of the sequence $\left\{\left(g_{k}, A_{k}\right)\right\}$ satisfy the linear independence constraint qualification. In this situation, $\left\{s_{k}\right\}$ is bounded away from zero, the penalty parameter $\nu_{k}$ is constant and $g_{k}$ is negative for all large indices $k$, and stationarity of problem (1.2) is obtained, i.e., $\nabla f_{k}+A_{k} \lambda_{k} \rightarrow 0$, where the multipliers are defined by $\lambda_{k}=\mu S_{k}^{-1} e$.

This theorem isolates two situations where the KKT conditions may not be satisfied in the limit, both of which are of interest. Outcome (i) is a case where, in the limit, there is no direction improving feasibility to first order. This indicates that finding a feasible point is a problem that a local method cannot always solve without a good starting point. In considering outcome (ii) we must keep in mind that in some cases the solution to problem (1.2) is a point where the linear independence constraint qualification fails, and which is not a KKT point. Thus outcome (ii) may be just as relevant to the problem as satisfying the KKT conditions.

The rest of the section is devoted to the proof of this theorem, which will be presented in a sequence of lemmas addressing in order all the statements in the theorem. It is 
convenient to work with the following multiple of the merit function $\phi$

$$
\tilde{\phi}(x, s ; \nu) \equiv \frac{1}{\nu} \phi(x, s ; \nu)=\frac{1}{\nu}\left(f(x)-\mu \sum_{i=1}^{m} \ln s^{(i)}\right)+\|g(x)+s\| \quad(s>0) .
$$

Since step 4 of Algorithm I requires that $\phi$ be reduced sufficiently at every new iterate, we have that

$$
\tilde{\phi}\left(x_{k}, s_{k} ; \nu_{k-1}\right) \leq \tilde{\phi}\left(x_{k-1}, s_{k-1} ; \nu_{k-1}\right)-\frac{\eta \operatorname{pred}_{k-1}}{\nu_{k-1}}
$$

and therefore

$$
\tilde{\phi}\left(x_{k}, s_{k} ; \nu_{k}\right) \leq \tilde{\phi}\left(x_{k-1}, s_{k-1} ; \nu_{k-1}\right)+\left(\frac{1}{\nu_{k}}-\frac{1}{\nu_{k-1}}\right)\left(f_{k}-\mu \sum_{i=1}^{m} \ln s_{k}^{(i)}\right)-\frac{\eta \operatorname{pred}_{k-1}}{\nu_{k-1}} .
$$

This indicates that the sequence $\left\{\tilde{\phi}\left(x_{k}, s_{k} ; \nu_{k}\right)\right\}$ is not necessarily monotone when $\nu_{k}$ is updated. To deal with this difficulty, we first establish that, under mild assumptions, the slack variables are bounded above.

Lemma 4.4. Assume that $\left\{f_{k}\right\}$ is bounded below and that $\left\{g_{k}\right\}$ is bounded. Then the sequence $\left\{s_{k}\right\}$ is bounded, which implies that $\left\{\phi\left(x_{k}, s_{k} ; \nu_{k}\right)\right\}$ is bounded below.

Proof. Let $\gamma$ be an upper bound for $-f_{k}$ and for $\left\|g_{k}\right\|$. Since

$$
\sum_{i=1}^{m} \ln s_{k}^{(i)} \leq m \ln \left\|s_{k}\right\|_{\infty} \leq m \ln \left\|s_{k}\right\|
$$

equation (4.1), the fact that the sequence $\left\{\nu_{k}\right\}$ is monotone non-decreasing, and the nonnegativity of $\operatorname{pred}_{k}$ give

$$
\tilde{\phi}\left(x_{k}, s_{k} ; \nu_{k}\right) \leq \tilde{\phi}\left(x_{0}, s_{0} ; \nu_{0}\right)+\left(\frac{1}{\nu_{0}}-\frac{1}{\nu_{k}}\right)\left(\gamma+\mu m \max _{0 \leq j \leq k} \ln \left\|s_{j}\right\|\right) .
$$

On the other hand, from the definition of $\tilde{\phi}$ and (4.2) we have that for any $k$,

$$
\tilde{\phi}\left(x_{k}, s_{k} ; \nu_{k}\right) \geq-\frac{1}{\nu_{k}}\left(\gamma+\mu m \ln \left\|s_{k}\right\|\right)+\left\|s_{k}\right\|-\left\|g_{k}\right\| .
$$

Now, consider the indices $l_{j}$ such that $\left\|s_{l_{j}}\right\|=\max _{k \leq l_{j}}\left\|s_{k}\right\|$. Then combining (4.3)-(4.4) for $k$ given by any such $l_{j}$ we obtain

$$
-\frac{1}{\nu_{l_{j}}}\left(\gamma+\mu m \ln \left\|s_{l_{j}}\right\|\right)+\left\|s_{l_{j}}\right\|-\left\|g_{l_{j}}\right\| \leq \tilde{\phi}\left(x_{0}, s_{0} ; \nu_{0}\right)+\left(\frac{1}{\nu_{0}}-\frac{1}{\nu_{l_{j}}}\right)\left(\gamma+\mu m \ln \left\|s_{l_{j}}\right\|\right),
$$

and thus

$$
\left\|s_{l_{j}}\right\| \leq \tilde{\phi}\left(x_{0}, s_{0} ; \nu_{0}\right)+\gamma+\frac{1}{\nu_{0}}\left(\gamma+\mu m \ln \left\|s_{l_{j}}\right\|\right)
$$


Since the ratio $(\ln \|s\|) /\|s\|$ tends to 0 when $\|s\| \rightarrow \infty$, relation (4.5) implies that $\left\{s_{l_{j}}\right\}$ must be bounded. By definition of the indices $l_{j}$ we conclude that the whole sequence $\left\{s_{k}\right\}$ is bounded.

Given that the slack variables are bounded above and that $f_{k}$ is bounded below, it is clear that we may redefine the objective function $f$ - by adding a constant to it - so that

$$
f_{k}-\mu \sum_{i=1}^{m} \ln s_{k}^{(i)} \geq 0
$$

at all iterates, and that this change does not affect the problem or the algorithm in any way. This positivity, the fact that $\nu_{k}$ is nondecreasing and (4.1) imply that

$$
\tilde{\phi}\left(x_{k}, s_{k} ; \nu_{k}\right) \leq \tilde{\phi}\left(x_{k-1}, s_{k-1} ; \nu_{k-1}\right)-\frac{\eta \operatorname{pred}_{k-1}}{\nu_{k-1}}
$$

for all $k$.

We can now show that our rule in step 5 of Algorithm I for determining the new slack variables, $s_{k+1}=\max \left(s_{k}+d_{s},-g_{k+1}\right)$, is such that the step between two successive iterates is still controlled by the trust radius $\Delta_{k}$.

Lemma 4.5. Assume that $\left\{f_{k}\right\}$ is bounded below, that $\left\{g_{k}\right\}$ is bounded, and that $g$ is Lipschitz continuous on an open set $X$ containing all the iterates $x_{k}$. Then there exists a positive constant $\gamma_{1}$ such that for all $k \geq 1$,

$$
\left\|\left(x_{k+1}, s_{k+1}\right)-\left(x_{k}, s_{k}\right)\right\| \leq \gamma_{1} \Delta_{k} .
$$

Proof. Clearly, $\left\|\left(x_{k+1}, s_{k+1}\right)-\left(x_{k}, s_{k}\right)\right\| \leq\left\|x_{k+1}-x_{k}\right\|+\left\|s_{k+1}-s_{k}\right\|$ and $\left\|x_{k+1}-x_{k}\right\|=$ $\left\|d_{x}\right\| \leq\|d\| \leq \Delta_{k}$.

Consider now the step in $s$. Let $\gamma_{s}^{\prime}>0$ be the bound on $\left\{s_{k}\right\}$ given by Lemma 4.4. For the components $i$ of $s$ such that $s_{k+1}^{(i)}-s_{k}^{(i)}=d_{s}^{(i)}$, one has

$$
\left|s_{k+1}^{(i)}-s_{k}^{(i)}\right| \leq\left\|d_{s}\right\| \leq \gamma_{s}^{\prime}\left\|S_{k}^{-1} d_{s}\right\| \leq \gamma_{s}^{\prime} \Delta_{k}
$$

For the other components,

$$
s_{k}^{(i)}-s_{k+1}^{(i)} \leq-d_{s}^{(i)} \leq\left\|d_{s}\right\| \leq \gamma_{s}^{\prime} \Delta_{k}
$$

and $s_{k+1}^{(i)}=-g_{k+1}^{(i)}$ so that, using the fact that $g_{k}+s_{k} \geq 0$ (when $\left.k \geq 1\right)$, one has

$$
s_{k+1}^{(i)}-s_{k}^{(i)}=-g_{k+1}^{(i)}+g_{k}^{(i)}-\left(g_{k}^{(i)}+s_{k}^{(i)}\right) \leq\left\|g_{k+1}-g_{k}\right\| \leq \gamma^{\prime}\left\|d_{x}\right\| \leq \gamma^{\prime} \Delta_{k},
$$

where $\gamma^{\prime}>0$ denotes the Lipschitz constant of $g$.

With the above two lemmas, we can begin to address convergence in the next result. It deals with the function $(x, s) \in \mathbf{R}^{n} \times \mathbf{R}_{+}^{m} \mapsto\|g(x)+s\|^{2}$, which is another measure of 
infeasibility for the original problem (1.1). Note that if the slack variables are scaled by $S_{k}^{-1}$, the gradient of this function with respect to the scaled variables is

$$
2\left(\begin{array}{c}
A(x) \\
S
\end{array}\right)(g(x)+s)
$$

We now show that the iterates generated by the algorithm approach stationarity for this infeasibility function $\|g(x)+s\|^{2}$.

Lemma 4.6. Assume that the sequences $\left\{g_{k}\right\},\left\{A_{k}\right\}$, and $\left\{B_{k}\right\}$ are bounded, that $\left\{f_{k}\right\}$ is bounded below, and that $g, A$, and $\nabla f$ are Lipschitz continuous on an open convex set $X$ containing all the iterates $x_{k}$. Then

$$
\lim _{k \rightarrow \infty}\left(\begin{array}{c}
A_{k} \\
S_{k}
\end{array}\right)\left(g_{k}+s_{k}\right)=0
$$

Proof. By the assumptions on $A$ and $g$, we have that the function

$$
\theta(x, s) \equiv\left\|\left(\begin{array}{c}
A(x) \\
S
\end{array}\right)(g(x)+s)\right\|
$$

is Lipschitz continuous on the open set $X \times \mathbf{R}_{+}^{m}$ containing all the iterates $\left(x_{k}, s_{k}\right)$, i.e., there is a constant $\gamma_{L}^{\prime}>0$ such that

$$
\left|\theta(x, s)-\theta\left(x_{l}, s_{l}\right)\right| \leq \gamma_{L}^{\prime}\left\|(x, s)-\left(x_{l}, s_{l}\right)\right\|,
$$

for any two points $(x, s)$ and $\left(x_{l}, s_{l}\right)$ in $X \times \mathbf{R}_{+}^{m}$.

Now consider an arbitrary iterate $\left(x_{l}, s_{l}\right)$ such that $\theta_{l} \equiv \theta\left(x_{l}, s_{l}\right) \neq 0$. We first want to show that in a neighborhood of this iterate all sufficiently small steps are accepted by Algorithm I. To do this define the ball

$$
\mathcal{B}_{l} \equiv\left\{(x, s):\left\|(x, s)-\left(x_{l}, s_{l}\right)\right\|<\theta_{l} /\left(2 \gamma_{L}^{\prime}\right)\right\} .
$$

By (4.7), for any $(x, s) \in \mathcal{B}_{l}$ we have that

$$
\theta(x, s) \geq \frac{1}{2} \theta_{l},
$$

which implies that $g(x)+s \neq 0$. We also know that the normal step satisfies (2.21), and have shown in Lemma 4.4 that $\left\{s_{k}\right\}$ is bounded. Using this, (2.36) and the boundedness assumptions on $\left\{A_{k}\right\}$ and $\left\{g_{k}+s_{k}\right\}$, we see that there is a constant $\gamma_{1}^{\prime}$ (independent of $k$ and $l)$, such that for any such iterate $\left(x_{l}, s_{l}\right)$ and any iterate $\left(x_{k}, s_{k}\right) \in \mathcal{B}_{l}$

$$
\operatorname{pred}_{k} \geq \rho \nu_{k} \operatorname{vpred}_{k} \geq \nu_{k} \gamma_{1}^{\prime} \theta_{l} \min \left(\xi \tau, \tilde{\Delta}_{k}, \theta_{l}\right) .
$$

Therefore, if $\Delta_{k}$ is sufficiently small we have

$$
\operatorname{pred}_{k} \geq \nu_{k} \gamma_{1}^{\prime} \theta_{l} \tilde{\Delta}_{k}
$$


Using this together with Lemma 3.1, and recalling the trust region constraint and the fact that $\tilde{\Delta}_{k}=\xi \Delta_{k}$, we obtain

$$
\frac{\left|\operatorname{ared}_{k}-\operatorname{pred}_{k}\right|}{\operatorname{pred}_{k}} \leq \frac{\gamma_{L}\left(\left(1+\nu_{k}\right)\left\|d_{x}\right\|^{2}+\left\|S_{k}^{-1} d_{s}\right\|^{2}\right)}{\nu_{k} \gamma_{1}^{\prime} \theta_{l} \tilde{\Delta}_{k}} \leq \frac{\gamma_{L}\left(1+\nu_{k}\right) \Delta_{k}^{2}}{\nu_{k} \gamma_{1}^{\prime} \theta_{l} \xi \Delta_{k}} .
$$

By making $\Delta_{k}$ sufficiently small we can ensure that the last term is less than or equal to $1-\eta$, and therefore for all $x_{k} \in \mathcal{B}_{l}$ and all such $\Delta_{k}$,

$$
\operatorname{ared}_{k} \geq \eta \operatorname{pred}_{k}
$$

implying (by (3.2)) acceptance of the step in Algorithm I.

Next we want to show that the rest of the iterates $\left\{x_{k}\right\}_{k>l}$ cannot remain in $\mathcal{B}_{l}$. We proceed by contradiction and assume that for all $k>l, x_{k} \in \mathcal{B}_{l}$ and therefore (4.9) holds for sufficiently small $\Delta_{k}$; this implies that there exists $\Delta^{0}>0$ such that $\Delta_{k}>\Delta^{0}$ for all $k>l$. This, together with (4.6) and (4.8) gives

$$
\tilde{\phi}_{k+1} \leq \tilde{\phi}_{k}-\frac{\eta}{\nu_{k}} \operatorname{pred}_{k} \leq \tilde{\phi}_{k}-\eta \gamma_{1}^{\prime} \theta_{l} \min \left(\xi \tau, \xi \Delta^{0}, \theta_{l}\right),
$$

where $\tilde{\phi}_{k} \equiv \tilde{\phi}\left(x_{k}, s_{k} ; \nu_{k}\right)$. Since the last term in the right hand side is constant, this relation implies that $\tilde{\phi}_{k} \rightarrow-\infty$, contradicting the conclusion of Lemma 4.4 that $\left\{\tilde{\phi}_{k}\right\}$ is bounded below. Therefore the sequence of iterates must leave $\mathcal{B}_{l}$ for some $k>l$.

Now let $\left(x_{k+1}, s_{k+1}\right)$ be the first iterate after $\left(x_{l}, s_{l}\right)$ that is not contained in $\mathcal{B}_{l}$. We must consider two possibilities. First, if there exists some $j \in[l, k]$ such that $\tilde{\Delta}_{j}>$ $\min \left(\xi \tau, \theta_{l}\right)$, then we have from (4.6) and (4.8) that

$$
\begin{aligned}
\tilde{\phi}_{k+1} & \leq \tilde{\phi}_{j+1} \\
& \leq \tilde{\phi}_{j}-\frac{\eta}{\nu_{j}} \operatorname{pred}_{j} \\
& \leq \tilde{\phi}_{j}-\eta \gamma_{1}^{\prime} \theta_{l} \min \left(\xi \tau, \theta_{l}\right) \\
& \leq \tilde{\phi}_{l}-\eta \gamma_{1}^{\prime} \theta_{l} \min \left(\xi \tau, \theta_{l}\right) .
\end{aligned}
$$

The other possibility is that for all $j \in[l, k], \tilde{\Delta}_{j} \leq \min \left(\xi \tau, \theta_{l}\right)$. In that case it follows from (4.6) and (4.8) that

$$
\begin{aligned}
\tilde{\phi}_{k+1} & \leq \tilde{\phi}_{l}-\sum_{j=l}^{k} \frac{\eta}{\nu_{j}} \operatorname{pred}_{j} \\
& \leq \tilde{\phi}_{l}-\sum_{j=l}^{k} \eta \gamma_{1}^{\prime} \theta_{l} \xi \Delta_{j} .
\end{aligned}
$$

Then, using Lemma 4.5 and the fact that $\left(x_{k+1}, s_{k+1}\right)$ has left the ball $\mathcal{B}_{l}$, whose radius is $\theta_{l} /\left(2 \gamma_{L}^{\prime}\right)$ give

$$
\sum_{j=l}^{k} \Delta_{j} \geq \frac{1}{\gamma_{1}}\left\|\left(x_{k+1}, s_{k+1}\right)-\left(x_{l}, s_{l}\right)\right\| \geq \frac{\theta_{l}}{2 \gamma_{L}^{\prime} \gamma_{1}}
$$


Substituting in (4.11) we obtain

$$
\tilde{\phi}_{k+1} \leq \tilde{\phi}_{l}-\eta \gamma_{1}^{\prime} \xi \theta_{l}^{2} /\left(2 \gamma_{L}^{\prime} \gamma_{1}\right)
$$

To conclude the proof note that since $\left\{\tilde{\phi}_{k}\right\}$ is decreasing and bounded below, we have that $\tilde{\phi}_{l} \rightarrow \tilde{\phi}_{*}$ for some infimum value $\tilde{\phi}_{*}$. Since $l$ was chosen arbitrarily, the fact that either (4.10) or (4.12) must hold at $\left(x_{l}, s_{l}\right)$ implies that $\theta_{l} \rightarrow 0$.

This result shows that $A_{k}\left(g_{k}+s_{k}\right) \rightarrow 0$ and $S_{k}\left(g_{k}+s_{k}\right) \rightarrow 0$. This is of course satisfied when $g_{k}+s_{k} \rightarrow 0$, that is when feasibility is attained asymptotically. However it can also occur when $g_{k}+s_{k} \nrightarrow \nrightarrow 0$ and the matrices $A_{k}$ and $S_{k}$ approach rank deficiency, a possibility we now investigate.

The procedure for updating the slack variables in step 5 of Algorithm I becomes important now. It ensures that

$$
g_{k}+s_{k} \geq g_{k}^{+} \geq 0
$$

holds at every iteration. Lemma 4.7 first uses this relation to show that the gradient $A_{k} g_{k}^{+}$ of the measure of infeasibility $x \mapsto \frac{1}{2}\left\|g(x)^{+}\right\|^{2}$ converges to zero. Then Lemma 4.7 shows that the case $g_{k}^{+} \not \rightarrow 0$ implies that the penalty parameters tend to infinity.

Lemma 4.7. Under the conditions of Lemma 4.6, $A_{k} g_{k}^{+} \rightarrow 0$. Moreover, if the sequence of iterates is not asymptotically feasible, i.e., if $g_{k}^{+} \not \rightarrow 0$, then the penalty parameters $\nu_{k}$ tend to infinity.

Proof. Let $\hat{A}, \hat{g}$, and $\hat{s}$ be limit points of the sequences $\left\{A_{k}\right\},\left\{g_{k}\right\}$, and $\left\{s_{k}\right\}$. Since these sequences are bounded, we only have to show that $\hat{A} \hat{g}^{+}=0$.

If $\hat{g}^{(i)} \geq 0$, the conditions $\hat{s} \geq 0$ and $\hat{S}(\hat{g}+\hat{s})=0$ (from Lemma 4.6) imply that $\hat{s}^{(i)}=0$. If $\hat{g}^{(i)}<0$, then from (4.13), $\hat{s}^{(i)} \neq 0$, which together with the equation $\hat{S}(\hat{g}+\hat{s})=0$ implies that $\hat{s}^{(i)}=-\hat{g}^{(i)}$. This shows that $\hat{g}+\hat{s}=\hat{g}^{+}$. Using the equation $\hat{A}(\hat{g}+\hat{s})=0$ (from Lemma 4.6), we obtain that $\hat{A} \hat{g}^{+}=0$, which proves the first part of the lemma.

If $g_{k}^{+} \not \nrightarrow 0,(4.13)$ implies that there is an index $i$ such that $\left(g_{k}+s_{k}\right)^{(i)} \not \rightarrow 0$. Since $S_{k}\left(g_{k}+s_{k}\right) \rightarrow 0$, there is a subsequence of indices $k$ such that $s_{k}^{(i)} \rightarrow 0$ and $\ln s_{k}^{(i)} \rightarrow-\infty$. Since $\left\{f_{k}\right\}$ is bounded below, this is incompatible with the decrease of $\phi\left(x_{k}, s_{k} ; \nu\right)$ for a fixed value of the penalty parameter $\nu>0$. Therefore $\nu_{k}$ is increased infinitely often, and because this is always at least by a constant factor, $\left\{\nu_{k}\right\}$ is unbounded.

This completes our discussion of the case when the sequence $\left\{x_{k}\right\}$ is not asymptotically feasible (item (i) of Theorem 4.3).

To continue the analysis we consider from now on only the case when feasibility is approached asymptotically. We will divide the analysis in two cases depending on whether the matrices $\left(A_{k}^{\top} S_{k}\right)$ lose rank or not. We use the notation $\sigma_{\min }(M)$ to denote the smallest singular value of a matrix $M$, and recall that in Definitions 4.2 we describe our notion of linear independence constraint qualification. 
Lemma 4.8. Suppose that the sequences $\left\{g_{k}\right\}$ and $\left\{A_{k}\right\}$ are bounded, that $\left\{f_{k}\right\}$ is bounded below, and that $g_{k}+s_{k} \rightarrow 0$. Then, either there is some bound $\hat{\sigma}>0$ such that

$$
\sigma_{\min }\left(\left(A_{k}^{\top} S_{k}\right)\right) \geq \hat{\sigma}
$$

for all $k$, or the sequence $\left\{\left(g_{k}, A_{k}\right)\right\}$ has a limit point $(\bar{g}, \bar{A})$ failing the linear independence constraint qualification. In the latter case, the penalty parameter $\nu_{k}$ goes to infinity.

Proof. If $\lim \inf \sigma_{\min }\left(\left(A_{k}^{\top} S_{k}\right)\right)=0$, there is a subsequence of iterates for which the smallest singular value of $\left(A_{k}^{\top} S_{k}\right)$ converges to 0 . Thus, since the sequence $\left\{\left(A_{k}, g_{k}, s_{k}\right)\right\}$ is bounded (by the assumptions), it has a limit point $(\bar{A}, \bar{g}, \bar{s})$ such that the matrix $\left(\bar{A}^{\top} \bar{S}\right)$ is rank deficient. Now $\bar{S}$ is diagonal, so that the set $\mathcal{I}=\left\{i: \bar{s}^{(i)}=0\right\}$ cannot be empty and the columns of $\bar{A}$ with index in $\mathcal{I}$ must be linearly dependent. Since we assume $g_{k}+s_{k} \rightarrow 0$, we have that $\bar{g}^{(i)}=0$ if and only if $i \in \mathcal{I}$, and it follows that the set $\left\{\bar{A}^{(i)}: \bar{g}^{(i)}=0\right\}$ is rank deficient.

Since for $i \in \mathcal{I}$, a subsequence of $\left\{s_{k}^{(i)}\right\}$ tends to zero, a subsequence of $\left\{-\ln s_{k}^{(i)}\right\}$ goes to infinity. Because $\left\{s_{k}\right\}$ is bounded and $\left\{f_{k}\right\}$ is bounded below, this is incompatible with the decrease of $\phi\left(x_{k}, s_{k} ; \nu\right)$, which would occur if $\nu_{k}$ were eventually constant. By the update rule for the penalty parameter, if $\nu_{k}$ is changed infinitely often then $\left\{\nu_{k}\right\}$ is unbounded.

For the rest of this section we will focus on the case where $\sigma_{\min }\left(\left(A_{k}^{\top} S_{k}\right)\right) \geq \hat{\sigma}>0$ for all $k$, which implies that $g_{k}+s_{k} \rightarrow 0$. First we will use this condition to bound the length of the normal step $v=\left(v_{x}, v_{s}\right)$ by a constant multiple of $\operatorname{vpred}_{k}\left(\operatorname{Lemma}_{4.9}\right)$; then we can use this relation to show that the sequence of penalty parameters $\nu_{k}$ is bounded (Lemma 4.10). Finally we will be able to show that the stationarity conditions for problem (1.2) are asymptotically satisfied (Lemma 4.11).

Lemma 4.9. Suppose that Assumptions 4.1 hold and that for some $\hat{\sigma}>0$,

$$
\sigma_{\min }\left(\left(A_{k}^{\top} S_{k}\right)\right) \geq \hat{\sigma}>0
$$

for all $k$. Then, there are positive constants $\gamma_{2}$ and $\gamma_{3}$ such that if $\left\|g_{k}+s_{k}\right\| \leq \gamma_{2}$,

$$
\left\|\left(v_{x}, S_{k}^{-1} v_{s}\right)\right\| \leq \gamma_{3} \operatorname{vpred}_{k}
$$

Proof. Recall that, by Lemma 2.2, the normal step must satisfy

$$
\left\|g_{k}+s_{k}\right\| \operatorname{vpred}_{k} \geq \frac{\gamma_{v}}{2}\left\|\left(\begin{array}{c}
A_{k} \\
S_{k}
\end{array}\right)\left(g_{k}+s_{k}\right)\right\| \min \left(\xi \tau, \tilde{\Delta}_{k}, \frac{\left\|\left(\begin{array}{c}
A_{k} \\
S_{k}
\end{array}\right)\left(g_{k}+s_{k}\right)\right\|}{\left\|\left(A_{k}^{\top} S_{k}\right)\right\|^{2}}\right) .
$$

We may assume that $g_{k}+s_{k} \neq 0$, for otherwise $\operatorname{vpred}_{k}=0, v_{k}=0$ (by the same argument as in the proof of Proposition 3.2), and (4.15) is trivially satisfied. 
Using (4.14) and letting $\bar{\sigma}_{1}=\sup _{k}\left\|\left(A_{k}^{\top} S_{k}\right)\right\|$, this implies

$$
\operatorname{vpred}_{k} \geq \frac{\gamma_{v} \hat{\sigma}}{2} \min \left(\xi \tau, \tilde{\Delta}_{k}, \frac{\hat{\sigma}\left\|g_{k}+s_{k}\right\|}{\bar{\sigma}_{1}^{2}}\right)
$$

Let us now assume that $\left\|g_{k}+s_{k}\right\|$ is strictly smaller than the constant $\xi \tau \bar{\sigma}_{1}^{2} / \hat{\sigma}$. Then the minimum in (4.16) cannot occur at $\xi \tau$, and (4.16) becomes

$$
\operatorname{vpred}_{k} \geq \frac{\gamma_{v} \hat{\sigma}}{2} \min \left(\tilde{\Delta}_{k}, \frac{\hat{\sigma}\left\|g_{k}+s_{k}\right\|}{\bar{\sigma}_{1}^{2}}\right) .
$$

We now consider two cases:

Case 1. Suppose $\left\|g_{k}+s_{k}\right\| \geq \frac{1}{2} \hat{\sigma} \tilde{\Delta}_{k}$. Then, using $\hat{\sigma} \leq \bar{\sigma}_{1}$ and the trust region constraint,

$$
\operatorname{vpred}_{k} \geq \frac{\gamma_{v} \hat{\sigma}}{2} \min \left(1, \frac{\hat{\sigma}^{2}}{2 \bar{\sigma}_{1}^{2}}\right) \tilde{\Delta}_{k} \geq \frac{\gamma_{v} \hat{\sigma}^{3}}{4 \bar{\sigma}_{1}^{2}}\left\|\left(v_{x}, S_{k}^{-1} v_{s}\right)\right\| .
$$

From this inequality, (4.15) follows immediately.

Case 2. Suppose

$$
\left\|g_{k}+s_{k}\right\| \leq \frac{1}{2} \hat{\sigma} \tilde{\Delta}_{k} .
$$

Consider an arbitrary vector $\bar{v} \in \mathbf{R}^{n+m}$ in the range of $\left(A_{k}^{\top} S_{k}^{2}\right)^{\top}$ that gives a lower objective in the normal subproblem (2.6) than $v=0$. We claim such a vector satisfies the constraints of (2.6) if $\left\|g_{k}+s_{k}\right\|$ is sufficiently small. Since $\bar{v}=\left(A_{k}^{\top} S_{k}^{2}\right)^{\top} w$ for some vector $w \in \mathbf{R}^{m}$,

or

$$
\left\|g_{k}+s_{k}\right\|^{2} \geq\left\|g_{k}+s_{k}+\left(A_{k}^{\top} S_{k}\right)\left(\begin{array}{c}
A_{k} \\
S_{k}
\end{array}\right) w\right\|^{2}
$$

$$
\left\|\left(A_{k}^{\top} A_{k}+S_{k}^{2}\right) w\right\|^{2} \leq-2\left(g_{k}+s_{k}\right)^{\top}\left(A_{k}^{\top} A_{k}+S_{k}^{2}\right) w .
$$

Using the Cauchy-Schwarz inequality, this implies that

$$
\left\|\left(A_{k}^{\top} A_{k}+S_{k}^{2}\right) w\right\| \leq 2\left\|g_{k}+s_{k}\right\|
$$

and by (4.14), it follows that

$$
\left\|\left(\bar{v}_{x}, S_{k}^{-1} \bar{v}_{s}\right)\right\|=\left\|\left(\begin{array}{c}
A_{k} \\
S_{k}
\end{array}\right) w\right\| \leq \frac{2}{\hat{\sigma}}\left\|g_{k}+s_{k}\right\| .
$$

Together, (4.18) and (4.19) imply $\bar{v}$ is within the trust region. In addition, for each slack variable $s^{(i)},(4.19)$ implies

$$
\left(S_{k}^{-1} \bar{v}_{s}\right)^{(i)} \geq-\left\|\left(\bar{v}_{x}, S_{k}^{-1} \bar{v}_{s}\right)\right\| \geq-\frac{2}{\hat{\sigma}}\left\|g_{k}+s_{k}\right\| \geq-\xi \tau,
$$

provided that $\left\|g_{k}+s_{k}\right\| \leq(\xi \tau \hat{\sigma}) / 2$. Thus $\bar{v}$ is feasible for $(2.6)$.

Now consider the problem (2.6) and its transformed equivalent (2.9). Since $\left(A_{k}^{\top} S_{k}\right)$ is of full rank there is a solution $\bar{u}$ to the equation $g_{k}+s_{k}+A_{k}^{\top} u_{x}+S_{k} u_{s}=0$, of minimum 
Euclidean norm, which is known to lie in the range of $\left(A_{k}^{\top} S_{k}\right)^{\top}$. Thus $\bar{v}=\left(\bar{u}_{x}, S_{k} \bar{u}_{s}\right)$ lies in the range of $\left(A_{k}^{\top} S_{k}^{2}\right)^{\top}$, and gives a value of zero for the objective of (2.6). By the above argument, if $\left\|g_{k}+s_{k}\right\|$ is sufficiently small, $\bar{v}$ is feasible for problem (2.6), and is therefore a solution to (2.6). Since $\bar{v}$ is a solution to (2.6) lying in the range of $\left(A_{k}^{\top} S_{k}^{2}\right)^{\top}$, the range space condition (2.11) implies that the normal step $v_{k}$ must also lie in the range of $\left(A_{k}^{\top} S_{k}^{2}\right)^{\top}$. This implies that, since $\operatorname{vpred}_{k}\left(v_{k}\right) \geq 0, v_{k}$ satisfies $(4.19)$, so that

$$
\left\|\left(v_{x}, S_{k}^{-1} v_{s}\right)\right\| \leq \frac{2}{\hat{\sigma}}\left\|g_{k}+s_{k}\right\| .
$$

Now recall that by (4.17) and (4.18),

$$
\operatorname{vpred}_{k} \geq \frac{\gamma_{v} \hat{\sigma}}{2} \min \left(\frac{2}{\hat{\sigma}}, \frac{\hat{\sigma}}{\bar{\sigma}_{1}^{2}}\right)\left\|g_{k}+s_{k}\right\|,
$$

which together with (4.21) implies (4.15).

We should note that if the Lagrange multipliers $\lambda_{k}$ are defined as the least squares solution to

$$
\left(\begin{array}{c}
\nabla f_{k}+A_{k} \lambda \\
S_{k} \lambda-\mu e
\end{array}\right)=0,
$$

then the boundedness of $\left\{\nabla f_{k}\right\},\left\{A_{k}\right\},\left\{s_{k}\right\}$, and (4.14) imply that the sequence $\left\{\lambda_{k}\right\}$ is bounded. The boundedness assumption on $B_{k}$ is now easy to enforce in this case, particularly if $B_{k}$ is defined as $\nabla_{x x}^{2} L\left(x_{k}, s_{k}, \lambda_{k}\right)$.

With the bound (4.15) on the normal step, in the case where $g_{k}+s_{k} \rightarrow 0$, we can show that the parameter $\nu_{k}$ eventually becomes fixed.

Lemma 4.10. Suppose that Assumptions 4.1 are satisfied, and that (4.15) holds for $k$ sufficiently large. Then, the sequence of penalty parameters $\left\{\nu_{k}\right\}$ is bounded. In addition, there exists an index $k_{1}$ and positive scalars $\bar{\nu}$ and $\gamma_{4}$, such that for all $k \geq k_{1}$,

$$
\nu_{k}=\bar{\nu}
$$

and

$$
\operatorname{pred}_{k}\left(d_{k}\right) \geq \gamma_{4} \operatorname{hpred}_{k}
$$

Proof. In step 3 of Algorithm I, $\nu_{k}$ is chosen to be sufficiently large such that

$$
\operatorname{pred}_{k}\left(d_{k}\right) \geq \rho \nu_{k} \operatorname{vpred}_{k}
$$

where, as in (2.37)-(2.38)

$$
\begin{aligned}
\operatorname{pred}_{k}\left(d_{k}\right)= & \nu_{k} \operatorname{vpred}_{k}+\operatorname{hpred}_{k} \\
& -\nabla f_{k}^{\top} v_{x}-\frac{1}{2} v_{x}^{\top} B_{k} v_{x}+\mu\left(e^{\top} S_{k}^{-1} v_{s}-\frac{1}{2} v_{s}^{\top} S_{k}^{-2} v_{s}\right) .
\end{aligned}
$$


We consider the terms in the second line of the above equation. By Assumptions 4.1, $\left\{\nabla f_{k}\right\},\left\{A_{k}\right\}$, and $\left\{B_{k}\right\}$ are all bounded. Note also that $\left\{\operatorname{vpred}_{k}\right\}$ is bounded, since by (2.14), $\operatorname{vpred}_{k} \leq\left\|g_{k}+s_{k}\right\|$, and this quantity is bounded as a consequence of Assumption 4.1 and Lemma 4.4. Therefore, using (4.15), there is a constant $\gamma_{1}^{\prime}>0$ such that

$$
-\nabla f_{k}^{\top} v_{x}-\frac{1}{2} v_{x}^{\top} B_{k} v_{x}+\mu\left(e^{\top} S_{k}^{-1} v_{s}-\frac{1}{2} v_{s}^{\top} S_{k}^{-2} v_{s}\right) \geq-\gamma_{1}^{\prime} \operatorname{vpred}_{k} .
$$

Hence from (4.24) the predicted decrease satisfies

$$
\operatorname{pred}_{k}\left(d_{k}\right) \geq \nu_{k} \operatorname{vpred}_{k}+\operatorname{hpred}_{k}-\gamma_{1}^{\prime} \operatorname{vpred}_{k} .
$$

Since $\operatorname{vpred}_{k}$ and $\operatorname{hpred}_{k}$ are nonnegative, we deduce from this inequality that condition (4.23) is satisfied if $\nu_{k} \geq \gamma_{1}^{\prime} /(1-\rho)$. Therefore, if $\nu_{k}$ becomes larger than $\gamma_{1}^{\prime} /(1-\rho)$, it will never be increased. This, together with the fact that whenever Algorithm I increases $\nu_{k}$ it does so by a constant factor, implies that after some iterate, $k_{1}$ say, $\nu_{k}$ will remain unchanged at some value $\bar{\nu}$.

Now consider (4.25) when $k \geq k_{1}$ and $\nu_{k}=\bar{\nu}$. If $\operatorname{hpred}_{k} \geq-2\left(\bar{\nu}-\gamma_{1}^{\prime}\right) \operatorname{vpred}_{k}$ then $\operatorname{pred}_{k}\left(d_{k}\right) \geq \frac{1}{2} \operatorname{hpred}_{k}$. Otherwise, $\operatorname{hpred}_{k}<-2\left(\bar{\nu}-\gamma_{1}^{\prime}\right) \operatorname{vpred}_{k}$ and it must be the case that $\bar{\nu}-\gamma_{1}^{\prime}<0$, in which case, by (4.23),

$$
\operatorname{pred}_{k}\left(d_{k}\right) \geq \frac{\rho \bar{\nu}}{2\left(\gamma_{1}^{\prime}-\bar{\nu}\right)} \operatorname{hpred}_{k}
$$

So (4.22) holds in either case.

Lemma 4.11. Suppose that Assumptions 4.1 hold and that the singular values of the matrices $\left(A_{k}^{\top} S_{k}\right)$ are bounded away from zero. Then,

(i) $\left\{s_{k}\right\}$ is bounded away from zero and $g_{k}$ is negative for all large $k$,

(ii) $\nabla f_{k}+\mu A_{k} S_{k}^{-1} e \rightarrow 0$.

Proof. By Lemma 4.6, $g_{k}+s_{k} \rightarrow 0$, and thus (4.15) eventually holds at all iterates. So, by Lemma 4.10, we have that $\nu_{k}=\bar{\nu}$ for all $k \geq k_{1}$. Since Algorithm I decreases the merit function at every iteration we have

$$
\phi\left(x_{k}, s_{k} ; \bar{\nu}\right) \leq \phi\left(x_{k_{1}}, s_{k_{1}} ; \bar{\nu}\right), \quad \text { for } k \geq k_{1} .
$$

Thus

$$
-\mu \sum_{i=1}^{m} \ln s_{k}^{(i)} \leq \phi\left(x_{k_{1}}, s_{k_{1}} ; \bar{\nu}\right)-f_{k}-\bar{\nu}\left\|g_{k}+s_{k}\right\| .
$$

Since we assume that $\left\{f_{k}\right\}$ is bounded below and because $\left\{s_{k}\right\}$ is bounded (Lemma 4.4), this implies that there is a vector $\bar{s}>0$ such that

$$
s_{k} \geq \bar{s}, \quad \text { for } k \geq 1 \text {. }
$$

Thus, because $g_{k}+s_{k} \rightarrow 0$, we have that $g_{k}<0$ for large $k$, proving (i). 
Next, recall that, by Lemma $2.3,\left(h_{x}, h_{s}\right)$ satisfies

$$
\operatorname{hpred}_{k} \geq \frac{\gamma_{h}}{2}\left\|p_{k}^{c}\right\| \min \left(\frac{\min \left(\hat{\Delta}_{k},(1-\xi) \tau\right)}{\left\|Z_{x}^{\top} Z_{x}+Z_{s}^{\top} S_{k}^{-2} Z_{s}\right\|^{1 / 2}}, \frac{\left\|p_{k}^{c}\right\|}{\left\|Z_{x}^{\top} B_{k} Z_{x}+\mu Z_{s}^{\top} S_{k}^{-2} Z_{s}\right\|}\right),
$$

where

$$
p_{k}^{c}=-Z_{x}^{\top}\left(\nabla f_{k}+B_{k} v_{x}\right)+\mu Z_{s}^{\top}\left(S_{k}^{-1} e-S_{k}^{-2} v_{s}\right),
$$

and where the null space basis matrix $Z_{k}=\left(Z_{x}^{\top} Z_{s}^{\top}\right)^{\top}$ is assumed to have singular values that are both bounded above and bounded away from zero. Since we have shown that all components of $s_{k}$ are bounded away from zero, it follows that $\left\{Z_{x}^{\top} Z_{x}+Z_{s}^{\top} S_{k}^{-2} Z_{s}\right\}$ is bounded. In addition since $\left\{B_{k}\right\}$ is bounded, $\left\{Z_{x}^{\top} B_{k} Z_{x}+\mu Z_{s}^{\top} S_{k}^{-2} Z_{s}\right\}$ is bounded. Hence, inequality (4.26) becomes

$$
\operatorname{hpred}_{k} \geq \gamma_{1}^{\prime}\left\|p_{k}^{c}\right\| \min \left(1, \hat{\Delta}_{k},\left\|p_{k}^{c}\right\|\right)
$$

for some positive constant $\gamma_{1}^{\prime}$.

To show that $\nabla f_{k}+\mu A_{k} S_{k}^{-1} e$ tends to zero, we relate this quantity to $p_{k}^{c}$. Note that the matrix $\left(I-A_{k}\right)^{\top}$ is a null space basis (see (2.27)), and that using the equivalence of null space bases we get

$$
\begin{aligned}
\nabla f_{k}+\mu A_{k} S_{k}^{-1} e & =\left(I-A_{k}\right)\left(\begin{array}{c}
\nabla f\left(x_{k}\right) \\
-\mu S_{k}^{-1} e
\end{array}\right) \\
& =\left(I-A_{k}\right) Z_{k}\left(Z_{k}^{\top} Z_{k}\right)^{-1} Z_{k}^{\top}\left(\begin{array}{c}
\nabla f\left(x_{k}\right) \\
-\mu S_{k}^{-1} e
\end{array}\right),
\end{aligned}
$$

for the chosen null space basis $Z_{k}$. By the boundedness of $A_{k}$ and of the singular values of $Z_{k}$ it follows from (4.28) that for some constant $\gamma_{2}^{\prime}$

$$
\left\|p_{k}^{c}\right\| \geq \gamma_{2}^{\prime}\left(\left\|q_{k}\right\|-\left\|v_{k}\right\|\right)
$$

for all $k$, where $q_{k}=\nabla f_{k}+\mu A_{k} S_{k}^{-1} e$.

We use a similar argument to that used in the proof of Lemma 4.6. To obtain a contradiction, suppose that $\theta=\frac{1}{4} \lim \sup _{k \rightarrow \infty}\left\|q_{k}\right\|$ is nonzero. Since $v_{k} \rightarrow 0$, we can find an iterate $\left(x_{l}, s_{l}\right)$ with arbitrarily large $l$ such that $\left\|q_{l}\right\|>3 \theta$ and such that $\left\|v_{k}\right\|<\theta$ for all $k \geq l$. Let $\bar{\gamma}_{L}$ be the Lipschitz constant for $q(x, s)=\nabla f(x)+\mu A(x) S^{-1} e$. Then any iterate $\left(x_{k}, s_{k}\right)$, with $k \geq l$, in the ball $\mathcal{B}=\left\{(x, s):\left\|(x, s)-\left(x_{l}, s_{l}\right)\right\|<\theta / \bar{\gamma}_{L}\right\}$, satisfies

$$
\left\|p_{k}^{c}\right\| \geq \gamma_{2}^{\prime}\left(\left\|q_{l}\right\|-\left\|q_{l}-q_{k}\right\|-\left\|v_{k}\right\|\right) \geq \gamma_{2}^{\prime}(3 \theta-\theta-\theta)=\gamma_{2}^{\prime} \theta .
$$

By Lemma 4.10 and (4.27), we have with $\gamma_{3}^{\prime}=\gamma_{4} \gamma_{1}^{\prime} \gamma_{2}^{\prime}$

$$
\operatorname{pred}_{k} \geq \gamma_{4} \operatorname{hpred}_{k} \geq \gamma_{3}^{\prime} \theta \min \left(1, \hat{\Delta}_{k}, \gamma_{2}^{\prime} \theta\right) \text {. }
$$

Therefore, for any iterate $\left(x_{k}, s_{k}\right)$ in $\mathcal{B}$, with $k \geq l$, if $\hat{\Delta}_{k}$ is sufficiently small we have

$$
\operatorname{pred}_{k} \geq \gamma_{3}^{\prime} \theta \hat{\Delta}_{k}
$$


Then by Lemma 3.1,

$$
\frac{\left|\operatorname{ared}_{k}-\operatorname{pred}_{k}\right|}{\operatorname{pred}_{k}} \leq \frac{\gamma_{L}(1+\bar{\nu}) \Delta_{k}^{2}}{\gamma_{3}^{\prime} \theta \hat{\Delta}_{k}} \leq 1-\eta
$$

for $\Delta_{k}$ sufficiently small, implying acceptance of the step.

Next we show that the rest of the iterates $\left\{\left(x_{k}, s_{k}\right)\right\}_{k \geq l}$ cannot remain in $\mathcal{B}$. To prove this by contradiction we assume that for all $k>l,\left(x_{k}, s_{k}\right) \in \mathcal{B}$ and therefore (4.30) holds for sufficiently small $\Delta_{k}$. This implies that there exists $\Delta^{0}>0$ such that $\hat{\Delta}_{k}>\Delta^{0}$ for all $k>l$. This, together with (4.29) and step 4 of Algorithm I, gives

$$
\phi_{k+1} \leq \phi_{k}-\eta \operatorname{pred}_{k} \leq \phi_{k}-\eta \gamma_{3}^{\prime} \theta \min \left(1, \Delta^{0}, \gamma_{2}^{\prime} \theta\right) .
$$

Since the second term in the right hand side is constant, this relation implies that $\phi_{k} \rightarrow$ $-\infty$, which gives a contradiction because Lemma 4.4 shows that $\left\{\phi_{k}\right\}$ is bounded below. Therefore the sequence of iterates must leave $\mathcal{B}$ for some $k>l$.

Now let $\left(x_{k+1}, s_{k+1}\right)$ be the first iterate after $\left(x_{l}, s_{l}\right)$ that is not contained in $\mathcal{B}$. We must consider two possibilities. First, if there exists some $j \in[l, k]$ such that $\hat{\Delta}_{j}>\min \left(1, \gamma_{2}^{\prime} \theta\right)$, then we have from (4.29) that

$$
\begin{aligned}
\phi_{k+1} & \leq \phi_{j+1} \\
& \leq \phi_{j}-\eta \operatorname{pred}_{j} \\
& \leq \phi_{j}-\eta \gamma_{3}^{\prime} \theta \min \left(1, \gamma_{2}^{\prime} \theta\right) \\
& \leq \phi_{l}-\eta \gamma_{3}^{\prime} \theta \min \left(1, \gamma_{2}^{\prime} \theta\right)
\end{aligned}
$$

The other possibility is that for all $j \in[k, l], \hat{\Delta}_{j} \leq \min \left(1, \gamma_{2}^{\prime} \theta\right)$. In that case, it follows from (4.29) and (2.24) that

$$
\begin{aligned}
\phi_{k+1} & \leq \phi_{l}-\eta \sum_{j=l}^{k} \operatorname{pred}_{j} \\
& \leq \phi_{l}-\eta \gamma_{3}^{\prime} \theta(1-\xi) \sum_{j=l}^{k} \Delta_{j} \\
& \leq \phi_{l}-\eta \gamma_{3}^{\prime} \gamma_{4}^{\prime}(1-\xi) \theta^{2}
\end{aligned}
$$

The last inequality follows from the fact that $\left(x_{k+1}, s_{k+1}\right)$ has left the ball $\mathcal{B}$, whose radius is $\theta / \bar{\gamma}_{L}$, so that, as at the end of Lemma $4.6, \sum_{j=l}^{k} \Delta_{j} \geq \gamma_{4}^{\prime} \theta$, for some constant $\gamma_{4}^{\prime}$.

Since the sequence $\left\{\phi_{k}\right\}$ is decreasing and bounded below, it converges. This is in contradiction with the fact that $l$ may be chosen arbitrarily large in (4.31) or (4.32), and the fact that $\theta \neq 0$. Therefore $q_{k} \rightarrow 0$.

Now we have established all points of our main convergence result, Theorem 4.3, which we restate and whose proof we now summarize.

Theorem 4.12. Suppose that Algorithm I is applied to the barrier problem (1.2) and that Assumptions 4.1 hold. Then, 
1) the sequence of slack variables $\left\{s_{k}\right\}$ is bounded,

2) $A_{k}\left(g_{k}+s_{k}\right) \rightarrow 0$ and $S_{k}\left(g_{k}+s_{k}\right) \rightarrow 0$.

Furthermore, one of the following three situations occurs.

(i) The sequence $\left\{x_{k}\right\}$ is not asymptotically feasible. In this situation, the iterates approach stationarity of the measure of infeasibility $x \mapsto\left\|g(x)^{+}\right\|$, meaning that $A_{k} g_{k}^{+} \rightarrow 0$, and the penalty parameters $\nu_{k}$ tend to infinity.

(ii) The sequence $\left\{x_{k}\right\}$ is asymptotically feasible, but the sequence $\left\{\left(g_{k}, A_{k}\right)\right\}$ has a limit point $(\bar{g}, \bar{A})$ failing the linear independence constraint qualification. In this situation also, the penalty parameters $\nu_{k}$ tend to infinity.

(iii) The sequence $\left\{x_{k}\right\}$ is asymptotically feasible and all limit points of the sequence $\left\{\left(g_{k}, A_{k}\right)\right\}$ satisfy the linear independence constraint qualification. In this situation, $\left\{s_{k}\right\}$ is bounded away from zero, the penalty parameter $\nu_{k}$ is constant and $g_{k}$ is negative for all large indices $k$, and stationarity of problem (1.2) is obtained, i.e., $\nabla f_{k}+A_{k} \lambda_{k} \rightarrow 0$, where the multipliers are defined by $\lambda_{k}=\mu S_{k}^{-1} e$.

Proof. Conclusion (1) was established in Lemma 4.4, and conclusion (2) in Lemma 4.6. In the case that $\left\{x_{k}\right\}$ is not asymptotically feasible $\left(g_{k}^{+} \not \rightarrow 0\right)$, it was shown in Lemma 4.7 that situation $(i)$ occurs. If $g_{k}^{+} \rightarrow 0$, it was shown in Lemma 4.8, Lemma 4.10, and Lemma 4.11 that either (ii) or (iii) must hold.

\section{Overall Algorithm}

In this section we consider the overall algorithm, in which Algorithm I is run for decreasing values of the barrier parameter $\mu$. We are not concerned here with conditions assuring a good rate of convergence, but consider only the global convergence properties of this algorithm.

Algorithm II. Choose an initial value $\mu_{1}>0$ for the barrier parameter, a reduction factor $a \in(0,1)$, and a sequence of stopping tolerances $\left\{\epsilon_{l}\right\}_{l \geq 1}$ that tends to zero. Choose an initial iterate $\left(x_{0}, s_{0}\right)$ and set $l=1$ and $k_{0}=0$.

1. Apply Algorithm I from the point $\left(x_{k_{l-1}}, s_{k_{l-1}}\right)$ until it finds a point $\left(x_{k_{l}}, s_{k_{l}}\right)$ satisfying

$$
\begin{gathered}
\left\|g_{k_{l}}+s_{k_{l}}\right\| \leq \epsilon_{l}, \\
\left\|\nabla f_{k_{l}}+A_{k_{l}} \lambda_{k_{l}}\right\| \leq \epsilon_{l},
\end{gathered}
$$

where $\lambda_{k_{l}}=\mu_{l} S_{k_{l}}^{-1} e$.

2. Choose $\mu_{l+1} \in\left(0, a \mu_{l}\right)$.

3. Increase $l$ by 1 , and go to step 1 .

All the iterates generated by this algorithm form a single sequence $\left\{\left(x_{k}, s_{k}\right)\right\}_{k \geq 0}$. The index $k_{l-1}(l \geq 1)$ labels the starting point of the $l$ th outer iteration, which ends at the point $\left(x_{k_{l}}, s_{k_{l}}\right)$. 
Theorem 5.1. Suppose that $\left\{\left(x_{k}, s_{k}\right)\right\}$ is generated by Algorithm II and that, for each barrier problem, Assumptions 4.1 hold. Then, one of the following two possible outcomes can occur.

(A) For some parameter $\mu_{l}$, either inequality (5.1) is never satisfied, in which case the stationarity condition for minimizing $x \mapsto\left\|g(x)^{+}\right\|$is satisfied in the limit, i.e., $A\left(x_{k}\right) g\left(x_{k}\right)^{+} \rightarrow 0$, or else $g_{k}+s_{k} \rightarrow 0$ but inequality (5.2) is never satisfied, in which case the sequence $\left\{\left(g_{k}, A_{k}\right)\right\}$ has a limit point $(\bar{g}, \bar{A})$ failing the linear independence constraint qualification.

(B) At each outer iteration l of Algorithm II, the inner algorithm succeeds in finding a pair $\left(x_{k_{l}}, s_{k_{l}}\right)$ satisfying (5.1)-(5.2). All limit points $\hat{x}$ of $\left\{x_{k_{l}}\right\}$ are feasible. Furthermore, if any limit point $\hat{x}$ of $\left\{x_{k_{l}}\right\}$ satisfies the linear independence constraint qualification, then the first order optimality conditions of the problem

$$
\begin{array}{cl}
\min _{x} & f(x) \\
\text { s.t. } & g(x) \leq 0
\end{array}
$$

hold at $\hat{x}$ : there exists $\hat{\lambda} \in \mathbf{R}^{m}$ such that

$$
\nabla \hat{f}+\hat{A} \hat{\lambda}=0, \quad \hat{g} \leq 0, \quad \hat{\lambda} \geq 0, \quad \hat{g}^{\top} \hat{\lambda}=0 .
$$

Proof. Suppose that, for some value of $\mu_{l}$, Algorithm II fails to find a point satisfying (5.1) and (5.2). This implies that Algorithm I generates an infinite sequence for problem (1.2) with $\mu=\mu_{l}$, but that outcome (iii) of Theorem 4.12 does not occur. Since Assumptions 4.1 hold this implies that, for that value of $\mu$, either outcome $(i)$ or (ii) of Theorem 4.12 occurs, which leads to conclusion $(A)$.

The only other possibility is that Algorithm II satisfies (5.1)-(5.2) for all $l \geq 1$. Let $\mathcal{L}$ be a subsequence of indices $l$, such that $x_{k_{l}} \rightarrow \hat{x}$ when $l \rightarrow \infty$ in $\mathcal{L}$. Since $0 \leq g_{k_{l}}^{+} \leq g_{k_{l}}+s_{k_{l}}$ and $g_{k_{l}}+s_{k_{l}} \rightarrow 0$, one has $\hat{g}=g(\hat{x}) \leq 0$ ( $\hat{x}$ is feasible) and $s_{k_{l}} \rightarrow \hat{s}=-\hat{g}$ when $l \rightarrow \infty$ in $\mathcal{L}$.

Now suppose that the linear independence constraint qualification holds at $\hat{x}$ and consider the set of indices

$$
\mathcal{I}=\left\{i: \hat{g}^{(i)}=0\right\}
$$

For $i \notin \mathcal{I}, \hat{g}^{(i)}<0$ and $\hat{s}^{(i)}>0$, so that $\lambda_{k_{l}}^{(i)}=\mu_{l} / s_{k_{l}}^{(i)} \rightarrow 0$ when $l \rightarrow \infty$ in $\mathcal{L}$. From this and $\nabla f_{k_{l}}+A_{k_{l}} \lambda_{k_{l}} \rightarrow 0$, we deduce that

$$
\nabla f_{k_{l}}+\sum_{i \in \mathcal{I}} \lambda_{k_{l}}^{(i)} \nabla g_{k_{l}}^{(i)} \rightarrow 0
$$

By the constraint qualification hypothesis, the vectors $\left\{\nabla \hat{g}^{(i)}: i \in \mathcal{I}\right\}$ are linearly independent, so that, by (5.3), the positive sequence $\left\{\lambda_{k_{l}}\right\}_{l \in \mathcal{L}}$ converges to some value $\hat{\lambda} \geq 0$. Now, it remains to take the limit in $\nabla f_{k_{l}}+A_{k_{l}} \lambda_{k_{l}}$ when $l \rightarrow \infty$ in $\mathcal{L}$ and to observe that $\hat{g}^{\top} \hat{\lambda}=0$. Therefore conclusion $(B)$ holds. 


\section{Final Remarks}

In this paper we have presented and analyzed a trust region method for solving the barrier problem (1.2). This is an optimization problem with nonlinear equality constraints, plus the implicit constraint $s>0$. Our strategy has been to use a well-developed algorithm for equality constrained optimization and enforce the constraint $s>0$ by means of the trust region and the barrier term. Another benefit of using a trust region is the ability of the method to deal with indefiniteness of the Hessian and near rank deficiency of the constraints.

The algorithmic framework given in $\S 1$ can be used to implement primal or primaldual interior point methods. In this paper we have focused on primal methods because they are easier to analyze and we have devoted much attention to their global convergence properties because the analysis provides important clues on how to design the algorithms. Computational experience with the primal interior point method is given in $[16,4]$; those papers also provide computational results with primal-dual methods.

Another question to be dealt with is how to ensure that a good rate of convergence is obtained. This requires, among other things, a careful strategy for updating the barrier parameter $\mu$ and deciding how accurately to solve the barrier subproblems [5]. We should also mention that since our merit function is non-differentiable, getting fast convergence may necessitate use of a second-order correction or a watch-dog strategy to avoid the Maratos effect. Our computational experience [18,4] indicates that use of a second-order correction can be an efficient strategy for this purpose.

Acknowledgements. The authors are thankful to Clovis Gonzaga and Sanjay Mehrotra for numerous discussions on interior point methods that help guide this work. The perceptive comments of the referee and associate editor are also gratefully acknowledged.

\section{References}

[1] G. Blanchon, J.-C. Dodu, A. Renaud, and M. Bouhtou (1996). "Implementation of a primal-dual interior-point method applied to the planning of reactive power compensation devices". Proceedings of the 12th Power Systems Computation Conference, August 19-23, 1996, Dresden.

[2] K.M. Anstreicher and J.-P. Vial (1994). "On the convergence of an infeasible primaldual interior-point method for convex programming". Optimization Methods and Software, Vol. 3, pp. 273-283.

[3] R.H. Byrd (1987). "Robust trust region methods for constrained optimization". Talk given at the SIAM Conference on Optimization, Houston, TX, 1987.

[4] R.H. Byrd, M.E. Hribar and J. Nocedal (1997). "An interior point method for large scale nonlinear programming", Report OTC-97/04, Optimization Technology Center, Northwestern University. 
[5] R.H. Byrd, G. Liu and J. Nocedal (1997). "On the Local Behavior of an Interior Point Method for Nonlinear Programming", in Numerical Analysis 1997, D.F. Griffiths and D.J. Higham, eds, pp.37-56, Addison Wesley Longman.

[6] R.H. Byrd, R.B. Schnabel, and G.A. Schultz (1987). "A trust region algorithm for nonlinearly constrained optimization". SIAM Journal on Numerical Analysis, Vol. 24 , pp. $1152-1170$.

[7] M.R. Celis, J.E. Dennis, and R.A. Tapia (1985). "A trust region strategy for nonlinear equality constrained optimization", in Numerical Optimization 1984, P.T. Boggs, R.H. Byrd, R.B. Schnabel, eds., SIAM, Philadelphia, pp. 71-82.

[8] T.F. Coleman and Y. Li (1993). "An interior trust region approach for nonlinear minimization subject to bounds", Tech. Rep. TR93-1342, Department of Computer Science, Cornell University, to appear in SIAM Journal on Optimization.

[9] J.E. Dennis, M. Heinkenschloss, and L.N. Vicente (1994). "Trust-region interior-point SQP algorithms for a class of nonlinear programming problems", Tech. Report TR9445, Department of Computational and Applied Mathematics, Rice University.

[10] A.S. El-Bakry, R.A. Tapia, T. Tsuchiya, and Y. Zhang (1995). "On the formulation and theory of the Newton interior-point method for nonlinear programming". $J$. Optim. Theory App. 89, pp. 507-545.

[11] R. Fletcher (1987). Practical Methods of Optimization (second edition). John Wiley \& Sons (New York).

[12] P. E. Gill, W. Murray, and M. H. Wright (1981). Practical Optimization. Academic Press (London).

[13] C.C. Gonzaga (1992). "Path-following methods for linear programming". SIAM Review, Vol. 34, pp. 167-224.

[14] S. Granville (1994). "Optimal reactive dispatch through interior point methods". IEEE Transactions on Power Systems, Vol. 9, pp. 136-146.

[15] J. Herskovits (1993). "An interior points technique for nonlinear optimization". Technical Report, COPPE, Federal University of Rio de Janeiro.

[16] M.B. Hribar (1997). "Methods for Large-Scale Nonlinear Programming and Nonlinear Systems of Equations", Ph.D. Dissertation, EECS Department, Northwestern University.

[17] F. Jarre and M. Saunders (1995). "A Practical Interior-point Method for Convex Programming". SIAM Journal on Optimization, Vol. 5, pp. 149-171.

[18] M. Lalee, J. Nocedal, and T. Plantenga (1993). "On the Implementation of an Algorithm for Large-Scale Equality Constrained Optimization". Northwestern University, EECS Dept., Rep. NAM 08, to appear in SIAM Journal on Optimization. 
[19] I.J. Lustig, R.E. Marsten, and D.F. Shanno (1994). "Interior point methods for linear programming: Computational state of the art". ORSA Journal on Computing, 6, pp. $1-14$.

[20] E.O. Omojokun (1991). "Trust region algorithms for optimization with nonlinear equality and inequality constraints", Ph.D dissertation, University of Colorado.

[21] E.R. Panier, A.L. Tits, and J.N. Herskovits (1988). "A QP-free, globally convergent, locally superlinearly convergent algorithm for inequality constrained optimization". SIAM Journal on Control and Optimization, Vol. 26, pp. 788-811.

[22] T.D. Plantenga (1995). "A trust region method for nonlinear programming based on primal interior point techniques", to appear in SIAM J. Scient. Computing.

[23] M.J.D. Powell (1975). "Convergence properties of a class of minimization algorithms", in Nonlinear Programming 2, O. Mangasarian, R. Meyer, S. Robinson, eds., pp. 1-27.

[24] M.J.D. Powell and Y. Yuan (1991). "A trust region algorithm for equality constrained optimization". Mathematical Programming, Series A, Vol. 49, pp. 189-211.

[25] T. Steihaug (1993). "The conjugate gradient method and trust regions in large scale optimization". SIAM Journal on Numerical Analysis, Vol. 20, pp. 626-637.

[26] A. Vardi (1985). "A trust region algorithm for equality constrained minimization: convergence properties and implementation". SIAM Journal on Numerical Analysis, Vol. 22, pp. 575-591.

[27] H. Yamashita (1992). "A globally convergent primal-dual interior point method for constrained optimization". Technical Report, Mathematical Systems Institute Inc., Tokyo, Japan. (Revised in March 1994)

[28] M. H. Wright (1992). "Interior methods for constrained optimization". Acta Numerica 1992, pp. 341-407.

[29] Y.-C. Wu, A.S. Debs, and R.E. Marsten (1994). "A direct nonlinear predictorcorrector primal-dual interior point algorithm for optimal power flows". IEEE Transactions on Power Systems, Vol. 9, pp. 876-883. 